Marquette University

e-Publications@Marquette

2-1-1997

EPR Studies on the Mono- and Dicobalt(II)Substituted Forms of the Aminopeptidase from Aeromonas proteolytica. Insight into the Catalytic Mechanism of Dinuclear Hydrolases

Brian Bennett

MarquetteUniversity, brian.bennett@marquette.edu

Richard C. Holz

Marquette University, richard.holz@marquette.edu

Accepted version. Journal of the American Chemical Society, Vol. 119, No. 8 (February 1997): 1923-1933. DOI. (C) 1997 American Chemical Society. Used with permission.

Brian Bennett and Richard C. Holz were affiliated with Utah State University at the time of publication. 


\title{
EPR Studies on the Mono- and Dicobalt(II)-Substituted Forms of the Aminopeptidase from Aeromonas proteolytica. Insight into the Catalytic Mechanism of Dinuclear Hydrolases
}

\author{
Brian Bennett \\ Department of Chemistry and Biochemistry, \\ Utah State University, \\ Logan, UT \\ Richard C. Holz \\ Department of Chemistry and Biochemistry, \\ Utah State University, \\ Logan, UT
}


NOT THE PUBLISHED VERSION; this is the author's final, peer-reviewed manuscript. The published version may be accessed by following the link in the citation at the bottom of the page.

\section{Abstract}

The structure and function of the prototypical dinuclear hydrolase, namely, the aminopeptidase from Aeromonas proteolytica (AAP), was probed by EPR spectroscopy of the mono- and dicobalt(II)-substituted derivatives. A new systematic protocol for the interpretation of Co(II) EPR spectra is described and the $S=3 / 2$ spin states of the Co(II)-substituted forms of the enzyme have been characterized. This protocol allows the simulation of line shape using theoretically allowed $g_{\text {eff }}$ values corresponding to an isotropic $g_{\text {real }}$ value. In addition, the gross distortion of EPR spectra of high-spin $S=3 / 2 \mathrm{Co}$ (II) ions has been investigated, and the effects of saturation on the line shapes and on simulation-derived spectral parameters are discussed. For [Co-(AAP)], a distinctive EPR signal was observed in which the hyperfine pattern due to ${ }^{59} \mathrm{Co}$ was not centered on the low-field absorption feature, and the signal could not be simulated as a single species. Subtraction of EPR spectra recorded at different temperatures revealed that two species were, in fact, present in samples of [Co-(AAP)]. The first species was a relatively featureless axial signal with $g_{\text {eff }}$ values of $5.75,4.50$, and 2.50 . These values correspond to an $M_{\mathrm{s}}=\left| \pm^{1} / 2\right\rangle$ ground-state transition with $g_{\text {real }}=2.57$ and $E / D=0.08$. The second species had $g_{\text {eff }}$ values of $6.83,2.95$, and 1.96 and exhibited a characteristic eight-line ${ }^{59} \mathrm{Co}$ hyperfine pattern with $A_{z}=7.2 \mathrm{mT}$. The observed ${ }^{59} \mathrm{Co}$ hyperfine lines were simulated in both line width as well as signal intensity for the first time. These parameters correspond to the $M_{\mathrm{s}}=$ $\left| \pm^{1 / 2}\right\rangle$ ground-state transition with $g_{\text {real }}=2.57$; however, the signal exhibited marked rhombicity $(E / D=0.28)$, consistent with a highly distorted tetrahedral $\mathrm{Co}$ (II) species. The possibility that the spectrum could be due to contributions from the $M_{\mathrm{s}}=\left| \pm^{1 / 2}\right\rangle$ and $M_{\mathrm{s}}=\left| \pm^{3} / 2\right\rangle$ doublets of a single spin system was investigated, but subtraction of spectra recorded at various temperatures clearly indicated that the features at $g=2.95$ and $g=1.96$ were correlated with the feature at $g=6.83$. In addition, at temperatures above $15 \mathrm{~K}$, the signal intensity rapidly decreases and the signal is lost. The EPR spectrum of [ $\mathrm{CoCo}(\mathrm{AAP})]$ reveals a relatively featureless signal that was simulated as a single species with $g_{\text {eff }(1,2,3)}$ values of $5.10,3.85$, and $2.19 ; M_{s}$ $=| \pm 1 / 2\rangle ; g_{\text {real }}=2.25 ; E / D=0.095$. The intensity of the observed signal for [CoCo(AAP)] corresponded to $0.13 \mathrm{spin} / \mathrm{mol}$ of $\mathrm{Co}(\mathrm{II})$. These data strongly suggest that the two Co(II) ions in the active site of AAP experience significant spin-spin interaction and are either antiferromagnetically or ferromagnetically coupled. Perpendicular mode EPR titration of apo-AAP with Co(II) revealed a low-field signal extending out of zero-field in samples with more than 1 equiv of Co(II) added. This type of EPR absorption is indicative of an integral spin system. Coincident with the appearance of the low-field perpendicular mode signal was the appearance of a parallel mode EPR signal with $g \sim 12$. These data represent the first definitive evidence for ferromagnetic coupling between two high-spin $S=3 / 2 \mathrm{Co}$ (II) ions in a dinuclear center. The effect of $\mathrm{pH}$, added peroxide, and the coordination of the competitive inhibitor 1-butaneboronic acid (BuBA) on the signal both confirm the origin of the signal and provide important mechanistic information for this novel dicobalt(II) active site cluster. Based on the present study and 
NOT THE PUBLISHED VERSION; this is the author's final, peer-reviewed manuscript. The published version may be accessed by following the link in the citation at the bottom of the page.

the available literature data, a detailed mechanism of action is proposed for AAP.

\section{Introduction}

Enzymes containing di- and trinuclear metal centers play important roles in nature such as the oxidation of organic molecules coupled to the reduction of dioxygen, hydrolysis chemistry, reduction of nitrogen oxides, and dioxygen transport. ${ }^{1,2}$ The least-explored subclass of these enzymes includes the di- and trinuclear metallohydrolases. ${ }^{3}$ Crystallographically characterized enzymes that fall into this group include phospholipase $\mathrm{C}^{4}{ }^{4}$ alkaline phosphatase ${ }^{5}$ inositol monophosphatase, ${ }^{6}$ DNA polymerase $1,{ }^{7}$ the ribonuclease $\mathrm{H}$ domain of HIV-1 reverse transcriptase, ${ }^{8} \mathrm{P} 1$ nuclease, ${ }^{9}$ urease, ${ }^{10}$ the purple acid phosphatases, ${ }^{11-13}$ and the aminopeptidases. ${ }^{14-17}$ These multinuclear hydrolases play critical roles in hydrolyzing some of the most important molecules in life such as DNA, phospholipids, and polypeptides. ${ }^{2,3,18-21}$ They are therefore key players in carcinogenesis, tissue repair, and protein degradation processes. Dinuclear metallohydrolases are also involved in the degradation of agricultural neurotoxins as well as several chemical warfare agents. ${ }^{22-24}$ In addition, several multinuclear metallohydrolases are members of a subclass of dinuclear metalloenzymes that have been shown to contain carboxylate rich coordination environments. Some of the members of this biologically important group of proteins include the crystallographically characterized R2 subunit of ribonucleotide reductase, ${ }^{25}$ methane monooxygenase, ${ }^{26}$ and rubrytherin. ${ }^{27}$

Metallohydrolases utilize as their native metal ions every firstrow transition metal ion from manganese to zinc except copper. One of these, the methionine aminopeptidase from Escherichia coli (MAP), requires $2 \mathrm{~g}$-atoms of $\mathrm{Co}$ (II) for activity. ${ }^{14,28}$ Thus, MAP represents a new class of metalloenzyme that is dependent on Co(II) ions for enzymatic activity. A second aminopeptidase, from Aeromonas proteolytica (AAP), has been shown to be hyperactive by a factor of 7.7 toward the substrate I-alanine-p-nitroanilide upon substitution of

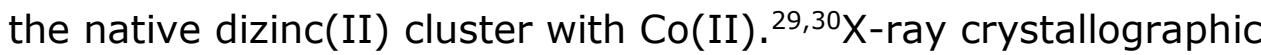
studies on MAP revealed a bis( $\mu$-carboxylato)dicobalt(II) active site with terminal carboxylates at each metal along with a histidine residue at the second $\mathrm{Co}(\mathrm{II})$ site. ${ }^{14}$ On the other hand, AAP possesses a $(\mu-$ 
aqua)( $\mu$-carboxylato)dizinc(II) core with one terminal carboxylate and one histidine residue at each metal site. ${ }^{17}$ Based on these data, both native and metal-substituted MAP and AAP, respectively, share structural similarities with one another as well as other physiologically important dinuclear metallohydrolases such as enolase, ${ }^{31,32}$ urease $^{10}$ and the functionally diverse phosphate ester hydrolases. 9,11,12,33-35

The active sites of several metalloenzymes have been probed by electron paramagnetic resonance (EPR) spectroscopy of their $S=3 / 2$ high-spin Co(II)-substituted derivatives. ${ }^{32,36-41}$ However, in almost all cases spectral parameters were extracted merely by observation rather than computer simulation, a notable exception being the work reported by Martinelli et al. ${ }^{39}$ Most of the reported spectra exhibit complicated line shapes that arise from saturation or from the presence of more than one species. ${ }^{39,42,43}$ As pointed out by Werth et al. ${ }^{41}$ further complications arise for high-spin $S=3 / 2 \mathrm{Co}$ (II) because the highest field $g$ feature may be undetectable. For spectra arising from the $\Delta m=\left| \pm^{3} / 2\right\rangle$ doublet for an almost axial species where $E / D$ approaches $0, g_{x, y}$ approaches 0 as does the transition probability for those resonances. Even when well-formed spectra are obtained, their interpretation in terms of coordination geometry is not trivial. Moreover, the suggestion that resolvable ${ }^{59} \mathrm{Co}$ hyperfine splitting is indicative of either a pentacoordinate or hexacoordinate ligand sphere is not a tenable hypothesis in light of the finding that the ratio of $\boldsymbol{A}_{(59 \mathrm{Co})}$ to $g_{\text {eff }}$ for $\mathrm{Co}$ (II) species of different coordination numbers is essentially constant. ${ }^{30,44}$ In principle, Co(II) coordination number can be inferred from the value of the zero-field splitting, $\Delta .^{45}$ In practice, estimation of $\Delta$ from the temperature dependence of the observed EPR signal has been shown to be unreliable; the estimation of $\Delta$ by this method is reliable only where relaxation occurs essentially by the Orbach process. However, delineation of the conditions under which the Orbach process dominates over the Raman and phonon-coupling relaxation regimes is difficult.

In an effort to gain insight into the structure and function of dinuclear hydrolases, we have examined the hyperactive Co(II)substituted aminopeptidase from Aeromonas proteolytica (AAP). We report herein EPR spectra of the mono- ([Co-(AAP)]) and dicobalt(II) $([\mathrm{CoCo}(\mathrm{AAP})])$ substituted enzymes. Based on these data, a new 
systematic protocol for the simulation of high-spin $S=3 / 2 \mathrm{Co}$ (II) EPR spectra is described. This protocol allows the simulation of line shape using theoretically allowed $g_{\text {eff }}$ values corresponding to an isotropic $g_{\text {real }}$ value. In addition, observed ${ }^{59} \mathrm{Co}$ hyperfine lines can be simulated in both line width as well as signal intensity for the first time. The gross distortion of EPR spectra of high-spin $S=3 / 2 \mathrm{Co}$ (II) ions, to our knowledge, has not been investigated in any detail, and very few reports mention that care must be taken to ascertain nonsaturating conditions. The effect of saturation on the line shapes of high-spin $S=$ $3 / 2$ Co(II) EPR spectra and on simulation-derived spectral parameters is discussed. In addition, the first definitive evidence for ferromagnetic coupling between two high-spin $S=3 / 2 \mathrm{Co}$ (II) ions in a dinuclear center using parallel mode EPR spectroscopy is presented. We have investigated the effects of $\mathrm{pH}$, added peroxide, and the coordination of the competitive inhibitor butaneboronic acid (BuBA) on the observed $S$ $=3$ signal in order to determine the nature of the interaction between the two Co(II) ions in substituted AAP. Based on the present study and the available literature data, a detailed mechanism of action is proposed for AAP.

\section{Materials and Methods}

Enzyme Purification. All chemicals used in this study were purchased commercially and were of the highest quality available. The aminopeptidase from Aeromonas proteolytica was purified from a stock culture kindly provided by Professor Céline Schalk. Cultures were grown according to the previously published procedure ${ }^{46}$ with minor modifications to the growth media, the details of which are presented elsewhere. ${ }^{47}$ Crude AAP is associated with two dark brown pigments that partition between the enzyme and Octyl Sepharose to different extents. These pigments are only completely removed from the enzyme during the ion exchange chromatography step. Both pigments have intense electronic absorption in the $250-$ to $300-\mathrm{nm}$ region. One of the pigments has an electronic absorption maximum at $400 \mathrm{~nm}$ and gives rise to an intense isotropic EPR resonance at $g=4.3$, indicative of it being associated with Fe(III) (data not shown). The $g=4.3$ EPR resonance can therefore be used as a sensitive test for pigment removal. The purified enzyme was found not to exhibit the $g=4.3$ EPR resonance. Because of the possibility that material with metal-binding 
potential is present in impure enzyme samples, great care was taken to ensure the protein was purified and pigment free. Denaturing gel electrophoresis indicated that the two resolvable activity-containing fractions from the Q-Sepharose column corresponded to the holoaminopeptidase and a processed form from which $13 \mathrm{~N}$-terminal amino acids have been cleaved ("AP1" and "AP2", respectively, in the nomenclature of Schalk et al. ${ }^{48}$ ). The enzyme was readily crystallized, a stringent test of homogeneity (C. DePaolo, D. Ringe, G. Petsko, B. Bennett and R. Holz, unpublished work), and was shown to be identical with the structure reported by Chevrier et al. ${ }^{17}$ The enzyme was stored at $77 \mathrm{~K}$ until needed.

AAP activity was measured by the method of Prescott and Wilkes ${ }^{46}$ as modified by Baker et al. ${ }^{49}$ In this assay, the hydrolysis of $0.5 \mathrm{mM}$ I-leucine-p-nitroanilide (10 mM Tricine, $\mathrm{pH} 8.0$, containing 0.1 $\mathrm{mM} \mathrm{ZnSO}_{4}$ ) was measured spectrophotometrically at $25^{\circ} \mathrm{C}$ by monitoring the formation of $p$-nitroaniline. The extent of hydrolysis was calculated by monitoring the increase in absorbance at $405 \mathrm{~nm}$ ( $\Delta \varepsilon_{405}$ value of $p$-nitroaniline of $10800 \mathrm{M}^{-1} \mathrm{~cm}^{-1}$ ). The specific activity of purified AAP with I-leucine-p-nitroanilide was typically found to be 120 units/mg of enzyme. One unit was defined as the amount of enzyme that releases $1 \mu \mathrm{mol}$ of $p$-nitroaniline at $25^{\circ} \mathrm{C}$ in $60 \mathrm{~s}$. The specific activity determined in this study is identical with that reported by Prescott and Wilkes. ${ }^{46}$ Enzyme concentrations were determined from the absorbance at $280 \mathrm{~nm}$ with the value $\varepsilon_{280}=41800 \mathrm{M}^{-1} \mathrm{~cm}^{-}$ 1.50 The accuracy of this value was checked by the Edelhoch method ${ }^{51-}$ ${ }^{53}$ using a 5:13:2 molar ratio mixture of $N$-acetyl-l-tryptophanamide, Gly-Tyr-amide, and I-cysteine, respectively, to model AAP. The molar absorptivity determined from this method, $\varepsilon_{280}=43950 \mathrm{M}^{-1} \mathrm{~cm}^{-1}$ for AAP solubilized in $6 \mathrm{M}$ guanidine hydrochloride, was in excellent agreement with the previously reported value by Prescott et al. ${ }^{50}$

Spectroscopic Measurements. All spectrophotometric measurements were performed on a Shimadzu UV-3101PC spectrophotometer equipped with a constant-temperature holder and a Haake (Type 423) constant-temperature circulating bath. Subtraction of the absorption spectrum of apo-AAP from that of the Co(II)substituted enzymes was performed using Shimadzu UV-3101 software. Low-temperature dual-mode EPR spectroscopy was 
performed using a Bruker ESP-300E spectrometer equipped with an ER 4116 DM dual-mode X-band cavity and an Oxford Instruments ESR900 helium flow cryostat. Background spectra recorded on an EPR tube containing buffer were aligned with and subtracted from experimental spectra as in earlier work. ${ }^{54,55}$ Signals due to oxygen were occasionally observed in both EPR modes. These signals routinely disappeared upon raising the temperature in the helium cryostat to $125 \mathrm{~K}$ for $5 \mathrm{~min}$ and recooling. All spectra were recorded at a modulation frequency of 100 $\mathrm{kHz}$ and modulation amplitude of $1.26 \mathrm{mT}(12.6 \mathrm{G})$ and with a sweep rate of $10 \mathrm{mT} \mathrm{s}^{-1}$. Parallel- and perpendicular-mode EPR spectra were recorded at microwave frequencies of about $9.37 \mathrm{GHz}$ and $9.65 \mathrm{GHz}$, respectively; precise microwave frequencies were recorded for individual spectra to ensure precise $g$ alignment. Other EPR running parameters are specified in the figure legends for individual samples. Enzyme concentrations for EPR were typically 1-2 mM. All buffers contained $20 \%$ isopropyl alcohol to prevent aggregation at high protein concentrations. Purified enzyme stored for up to 2 weeks at $4^{\circ} \mathrm{C}$ in Hepes buffer, $\mathrm{pH} 7.5$, containing $20 \%$ (by volume) isopropyl alcohol, showed no measurable decrease in activity. Computer techniques for resolution enhancement, sometimes used to resolve ${ }^{59} \mathrm{Co}$ hyperfine structure, have been used only where explicitly stated and have been described in detail elsewhere. ${ }^{56}$

Cobalt(II)-substituted AAP was prepared from the purified enzyme by a method similar to that of Prescott et al. ${ }^{30}$ Briefly, AAP was dialyzed for $72 \mathrm{~h}$ at $4^{\circ} \mathrm{C}$ against $10 \mathrm{mM}$ 1,10-phenanthroline monohydrochloride in $50 \mathrm{mM}$ Hepes buffer, $\mathrm{pH}$ 7.5, then exhaustively dialyzed against Chelex-treated Hepes buffer. Metal insertion was effected by direct addition of a solution of $\mathrm{CoCl}_{2}$ followed by a 30-min incubation period at $20-25^{\circ} \mathrm{C}$. Excess $\mathrm{Co}$ (II) was removed by successive dilution and concentration of [Co-(AAP)] or [ $\mathrm{CoCo}(A A P)]$ in an Amicon Centricon-10 microconcentrator under anaerobic conditions. In titrations of AAP with Co(II), 2 to $10 \mu \mathrm{L}$ of 8 to $32 \mathrm{mM} \geq 99.999 \%$ $\mathrm{CoCl}_{2}$ (Strem Chemicals, Newburyport, MA) was added to $200 \mu \mathrm{L}$ of enzyme solution in an EPR tube using a method described earlier to ensure rapid mixing. ${ }^{57} \mathrm{Co}$ (II)-substituted forms were assayed for activity with I-leucine- $p$-nitroanilide and I-alanine- $p$-nitroanilide $\left(\Delta \varepsilon_{405}\right.$ value of $p$-nitroaniline of $10800 \mathrm{M}^{-1} \mathrm{~cm}^{-1}$ ) and were entirely in agreement with those reported earlier. ${ }^{29,30}$ For example, the activities 
of Co(II)-substituted AAP with I-leucine $p$-nitroanilide and I-alanine $p$ nitroanilide were 24 and 0.9 units/mg, respectively, compared to the native dizinc(II) AAP enzyme which had specific activities of 120 and 0.2 units/mg, respectively. In agreement with earlier work, ${ }^{30}$ the enzyme was found to attain $\sim 80 \%$ of the native activity upon the addition of only 1 equiv of $\mathrm{Co}$ (II) or $\mathrm{Zn}(\mathrm{II})$.

Electronic absorption spectra were recorded for [Co-(AAP)] and $[\mathrm{CoCo}(\mathrm{AAP})]$, and the component due to apo-AAP was subtracted. The addition of one $\mathrm{Co}$ (II) ion to AAP provides a visible absorption spectrum with a band at $525 \mathrm{~nm}\left(\varepsilon_{525} \sim 50 \mathrm{M}^{-1} \mathrm{~cm}^{-1}\right)$. Addition of a second equivalent of $\mathrm{Co}$ (II) increases the absorption intensity at 525 $\mathrm{nm}$ to $\sim 90 \mathrm{M}^{-1} \mathrm{~cm}^{-1}$. The addition of more than 2 equiv of $\mathrm{Co}$ (II) does not alter the intensity or position of the observed absorption band. The added absorptivity $\left(\Delta \varepsilon_{525}\right.$ of $\sim 40 \mathrm{M}^{-1} \mathrm{~cm}^{-1}$ ) appears to be due to a broad underlying protein absorption band that is not due to $\mathrm{Co}$ (II) absorption. A similar broad absorption band was observed for the Co(II)-substituted R2 subunit of ribonucleotide reductase ${ }^{46}$ and is also evident in several Co(II) model complexes. ${ }^{47}$ Therefore, the increase in absorption at $525 \mathrm{~nm}$ due to the second $\mathrm{Co}$ (II) ion is $\sim 20 \mathrm{M}^{-1} \mathrm{~cm}^{-1}$. The molar absorptivities and absorption maxima are consistent with the first $\mathrm{Co}$ (II) ion residing in a tetrahedral coordination environment while the second $\mathrm{Co}$ (II) ion is octahedral. These spectra are identical with those recorded by Prescott and co-workers and are supported by magnetic circular dichroism spectra. ${ }^{30}$

\section{Computer Simulations of High-Spin $\mathrm{Co}$ (II) $\mathbf{S}=\mathbf{3} / \mathbf{2}$ EPR}

Spectra. EPR simulation of a single $S=3 / 2$ high-spin Co(II) species was carried out by treating the system as an effective $S=1 / 2$ system since Co(II) ions in either tetrahedral or octahedral ligand fields typically exhibit extensive spin-orbit coupling. The simulation program used was EPRSim XOP for Igor Pro by John Boswell (Oregon Graduate Institute) and is an adaptation of the program QPOW. ${ }^{59-61} \mathrm{~g}_{\text {real }}$ values and rhombicities $(E / D)$ were extracted from the $g_{\text {eff }}$ values obtained by simulation as follows: for high-spin $\mathrm{Co}(\mathrm{II})$, an $S=3 / 2$ system, the observed resonance positions, $g_{\operatorname{eff}(x, y, z)}$, are largely dictated by the zero-field splitting, $\Delta$, which is given by:

$$
\Delta \equiv 2 D \sqrt{\left[1+3(E / D)^{2}\right]}
$$

Journal of the American Chemical Society, Vol 119, No. 8 (February 26, 1997): pg. 1923-1933. DOI. This article is (C) American Chemical Society and permission has been granted for this version to appear in e-Publications@Marquette. American Chemical Society does not grant permission for this article to be further copied/distributed or hosted elsewhere without the express permission from American Chemical Society. 
where $D$ and $E$ are the axial and rhombic zero-field splitting parameters, respectively. The relationships between the $g_{\text {eff }(x, y, z)}$ values, $E / D$ and the corresponding $g_{\text {real }(x, y, z)}$ values has been shown ${ }^{62}$ to be:

$$
\begin{gathered}
g_{\mathrm{eff}(x)}=g_{\text {real }(x)}\left(1 \pm\left\{[1+3(E / D)] / \sqrt{\left[1+3(E / D)^{2}\right]}\right\}\right) \\
g_{\mathrm{eff}(y)}=g_{\mathrm{real}(y)}\left(1 \pm\left\{[1-3(E / D)] / \sqrt{\left[1+3(E / D)^{2}\right]}\right\}\right) \\
g_{\text {eff }(s)}=g_{\text {real }(s)}\left(1 \pm\left\{(-2) / \sqrt{\left[1+3(E / D)^{2}\right]}\right\}\right)
\end{gathered}
$$

From these relationships $g_{\text {real }(x, y, z)}$ and $E / D$ can be determined from $g_{\text {eff }(x, y, z)}$. In the present study $g_{\text {real }}$ and $E / D$ for each species simulated were determined by matching the output of a computer program, Rhombogram, kindly provided by Professor W. R. Hagen, to the $g_{\text {eff }}$ tensor derived by simulation as a function of $g_{\text {real }(x, y, z)}$ and $E / D$. As a consequence of the approach taken, two sets of $g_{\text {eff }}$ values were obtained; the $g_{\text {eff }}$ values obtained by simulation of the line shape are given followed in parentheses by the closest theoretically allowed values that satisfy eq 2-4 for an isotropic $g$ tensor. The match of these two sets of $g$ values is thus a quantitative indicator of the quality of the simulation. When experimental spectra contained two or more signals due to either different species or resonances from more that one accessible doublet, individual species were resolved by difference spectroscopy. Difference spectra were obtained by recording EPR data on samples under different spectral conditions such as different temperatures, microwave powers, or samples differing slightly in their relative amounts of the contributing species. ${ }^{57,58}$

\section{Results and Discussion}

Simulation of $\boldsymbol{S}=\mathbf{3} / \mathbf{2}$ High-Spin Co(II)-AAP EPR Spectra. Upon addition of 1 equiv of Co(II) to apo-AAP ([Co-(AAP)], a distinctive EPR signal was observed (Figure $1 \mathrm{~A}$ ). The hyperfine pattern due to ${ }^{59} \mathrm{Co}$ was not centered on the low-field absorption feature, and the signal could not be simulated as a single species. Similar complex 
signals from related enzymes have been reported; see, for example, the EPR spectra of cobalt-substituted enolase ${ }^{32}$ and the $[\mathrm{Zn}(\mathrm{c}) \mathrm{Co}(\mathrm{n})]$ form of horse liver alcohol dehydrogenase. ${ }^{41}$ However, no explanation of this phenomenon has been presented to date except for the case where magnetic ordering of $\mathrm{Co}$ (II) complexes gives rise to singlecrystal-like EPR spectra that resemble those observed for [Co-(AAP)]. ${ }^{47}$ In the present case, the freezing of magnetically dilute samples prior to exposure to a large magnetic field would preclude magnetic ordering of the $\mathrm{Co}$ (II) ions. Therefore, it was hypothesized that the observed signal arose from more than one species. Subtraction of EPR spectra of [Co-(AAP)] recorded at various temperatures or microwave powers revealed that the signal was, in fact, made up of two components. The first species (Figure $1, \mathrm{C}$ and D) was a relatively featureless axial signal with $g_{\text {eff }}$ values of 5.75 (5.76), 4.50 (4.48), and 2.50 (2.52) (the $g_{\text {eff }}$ values given are those that gave the best simulation, whereas those in parentheses are the closest allowed by theory; see Materials and Methods). These values correspond to an $M_{\mathrm{s}}$ $=| \pm 1 / 2\rangle$ ground-state transition with $g_{\text {real }}=2.57$ and $E / D=0.08$. The second species (Figure 1, E and F) had $g_{\text {eff }}$ values of 6.83 (6.83), 2.95 (2.94), and 1.96 (2.05) and exhibited a characteristic eight-line ${ }^{59} \mathrm{Co}$ hyperfine pattern with $A_{z}=7.2 \mathrm{mT}$ (Figure 1E). These parameters correspond to the $M_{\mathrm{s}}=| \pm 1 / 2\rangle$ ground-state transition with $g_{\text {real }}=$ 2.57; however, the signal exhibited marked rhombicity $(E / D=0.28)$ indicative of an asymmetric tetrahedral $\mathrm{Co}$ (II) ion, consistent with electronic absorption data. The possibility that the spectrum could be due to contributions from the $M_{\mathrm{s}}=| \pm 1 / 2\rangle$ and $M_{\mathrm{s}}=| \pm 3 / 2\rangle$ doublets of a single spin system was investigated, but good simulations could not be obtained and difference analyses of experimental spectra clearly correlated the features at $g=2.95$ and $g=1.96$ with that at $g$ $=6.83$. Theory dictates that these two lower $g$ values would be significantly less than unity and be barely observable if arising from an $M_{\mathrm{s}}=| \pm 3 / 2\rangle$ doublet. 


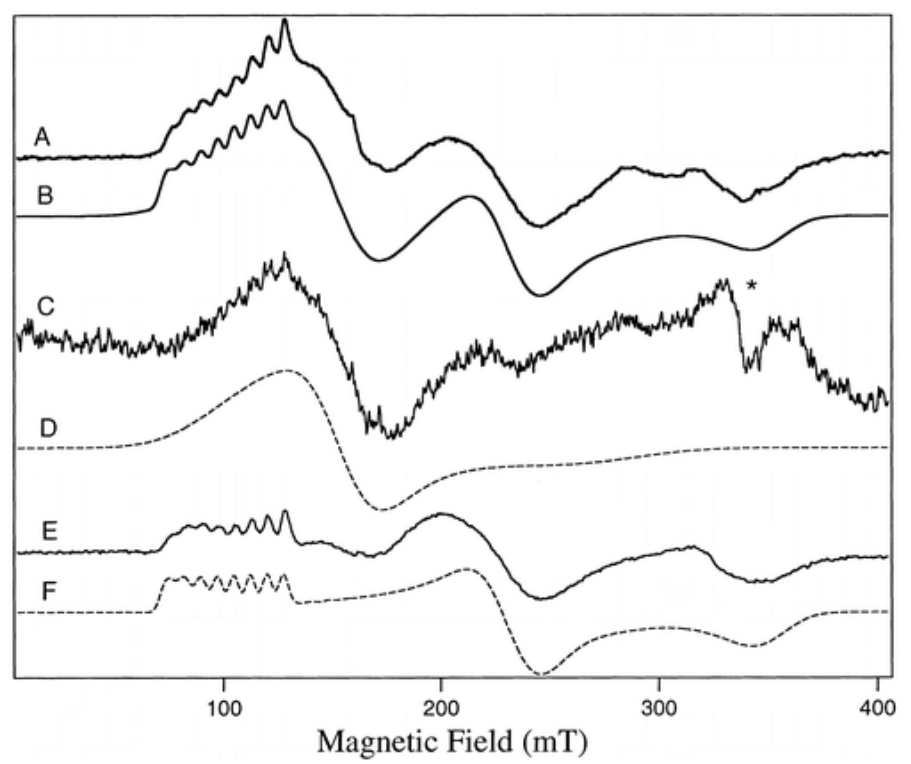

Figure 1 EPR spectra of [Co-(AAP)]: (A) EPR spectrum of [Co-(AAP)] recorded at 10 $\mathrm{K}, 0.2 \mathrm{~mW}$ microwave power; $(B)$ simulation of $A$ obtained by summing the individual simulations of $D$ and $F$; (C) EPR spectrum of an isotropic species obtained by subtraction of $E$ from A; (D) simulation of $C$ with $g_{\text {eff }}$ values of $5.75,4.50$, and 2.50; (E) EPR spectrum of the rhombic species obtained by subtracting the spectrum of [Co(AAP)] at $10 \mathrm{~K}, 2 \mathrm{~mW}$ microwave power (not shown) from $\mathrm{A} ;(\mathrm{F})$ simulation of $E$ with $g_{\text {eff }}$ values $6.83,2.95$, and $1.96, A_{z}=7.2 \mathrm{mT}$.

Simulation of the hyperfine lines observed for the second species found in the [Co-(AAP)] EPR spectrum, including their intensity variation, was achieved by inclusion of both $g$ strain and $g$-dependent $A$ strain in the simulation (Figure 2). While the computer program allowed for inclusion of $g$ strain, $A$ strain, and $g$ strain-A strain correlations, $\varepsilon$, the simulations were obtained by manually summing a number of spectra, shown in Figure 2C, with $g_{z}=6.83, \Delta g_{z}=0.18, A_{z}$ $=7.2 \mathrm{mT}, \Delta A_{z}=1.2 \mathrm{mT}$ with $g_{z}$ and $\Delta A$ fully correlated. This approach has the advantage of illustrating the effect of $g$ strain and $A$ strain on the spectrum as shown in Figure 2. 


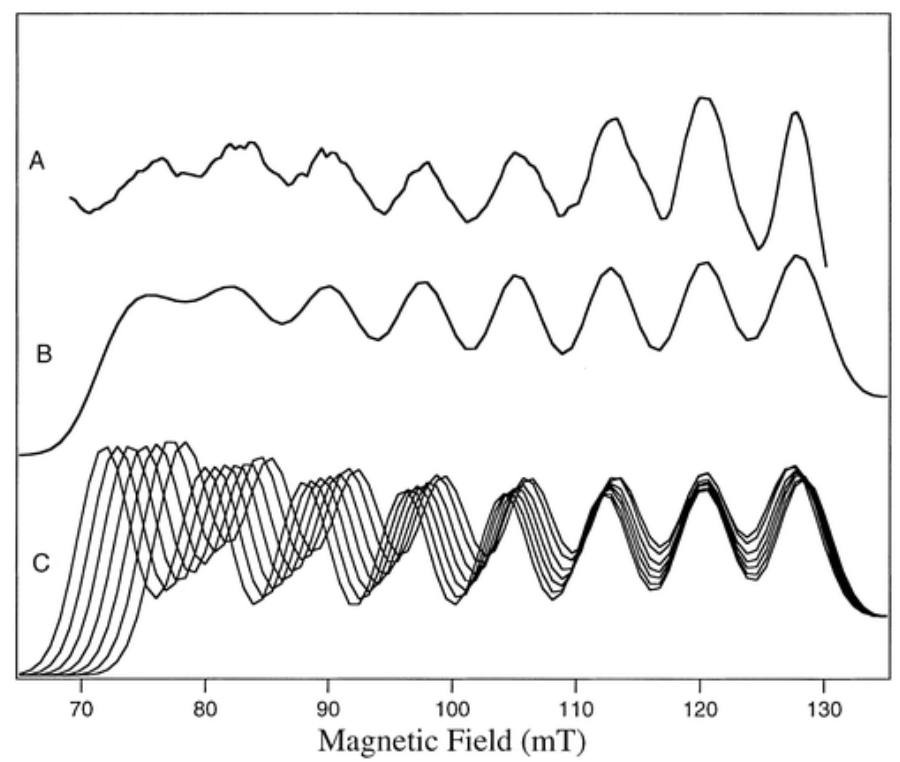

Figure 2 Simulation of the line shape of the hyperfine pattern of the EPR spectrum of [Co-(AAP)] due to ${ }^{59} \mathrm{Co}:(A)$ low-field region of the EPR spectrum of [Co-(AAP)] recorded at $10 \mathrm{~K}, 0.2 \mathrm{~mW}$ microwave power (underlying broad EPR absorption due to a second species was removed by subtraction of a third-order polynomial from the experimental spectrum); (B) simulation of A including $g$ strain $\left(g_{z}=6.83, \Delta g_{z}=0.18\right)$ and $g$-correlated $A$ strain $\left(A_{z}=7.2 \mathrm{mT}, \Delta A_{z}=1.2 \mathrm{mT}, \varepsilon=1\right)$; (C) graphical representation of the effects of $g$ and $A$ strain (the $g$ tensors of the Co(II) ions in the sample have slightly different orientations leading to slightly different $g$ values and $A$ values as shown). Summation of these spectra leads to the experimentally observed spectrum (A). Individual spectra shown in $C$ are simulations using $g_{z}=6.74,6.77$, $6.80,6.83,6.86,6.89,6.92$ and $A_{z}=6.6,6.8,7.0,7.2,7.4,7.6,7.8 \mathrm{mT}$, respectively.

The possibility that the featureless axial signal observed in [Co. (AAP)] spectra may be due to free $\mathrm{Co}(\mathrm{II})$ in buffer was also examined. A spectrum of $\mathrm{CoCl}_{2}$ in Hepes buffer at $\mathrm{pH} 7.5$ was recorded under conditions identical with those of [Co-(AAP)] and was found to exhibit a broad isotropic signal with markedly different EPR parameters compared to those of [Co-(AAP)]. Simulation of this spectrum provided $g_{\text {eff }}$ values of 5.08 (5.07), 4.04 (4.04), and 2.25 (2.25). These values correspond to an $M_{\mathrm{s}}=| \pm 1 / 2\rangle$ ground-state transition with $g_{\text {real }}=2.29$ and $E / D=0.08$. The EPR absorption of free Co(II) is also unusually broad and extends to zero field. Furthermore, an EPR spectrum of [Co$(A A P)]$ recorded at a $\mathrm{pH}$ value of 10.0 is primarily due to the second, more rhombic $(E / D=0.28)$ signal. The X-ray crystal structure of AAP shows that a single oxygen atom bridges between the two $\mathrm{Zn}$ (II) ions in the native enzyme, ${ }^{17,63}$ and kinetic studies indicate that this bridging water/hydroxide is only partially deprotonated at $\mathrm{pH} 7.5 .47$ Therefore, one possible explanation for the presence of two species would be the 
existence of a bridging water molecule that is in equilibrium with a bridging hydroxide. An alternative explanation would be that the two species observed in the EPR spectrum of [Co-(AAP)] may be the result of an equilibrium mixture of a four- and five-coordinate species. However, five-coordinate high-spin Co(II) ions exhibit little or no spin-orbit coupling so the electron spin lifetimes are long and EPR signals would be expected at high temperatures. Therefore, the $\mathrm{pH}$ dependence observed in the EPR spectrum of [Co-(AAP)] appears to be due to the deprotonation of a bridging water moiety to an hydroxide.

The EPR spectrum of [Co_(AAP)] was observable only under a very narrow range of EPR operating parameters at low temperatures, consistent with the assignment of the transitions to the ground-state doublet. Figure 3 shows how the ideal temperature-normalized signal intensity varies with temperature. The rather similar temperature dependence of both of the contributing signals is further evidence that they arise from separate species, both exhibiting transitions in the $M_{\mathrm{s}}$ $=| \pm 1 / 2\rangle$ doublet, rather than from $M_{s}=| \pm 1 / 2\rangle$ and $M_{s}=| \pm 3 / 2\rangle$ transitions in the same spin system. Figure 4 shows the microwave power saturation characteristics of the signal at $4 \mathrm{~K}$. The absolute signal intensity was greatest at $4 \mathrm{~K}$, but as can be seen in Figure 4 the onset of saturation occurred at even rather modest microwave powers. The lowest power at which sufficient signal intensity could be obtained in a reasonable time for routine work, $0.2 \mathrm{~mW}$, provided a signal that was slightly saturated. At this microwave power, this signal follows $1 / T$ dependence over a very narrow temperature range (Figure 3). Above $15 \mathrm{~K}$, relaxation broadening sets in very rapidly and the signal is lost. At temperatures below $10 \mathrm{~K}$ the onset of saturation occurs. The lack of a clear range of temperatures over which $1 / T$ intensity dependence can be observed at microwave powers high enough to give an observable EPR signal suggests that the signal cannot be observed under strictly nonsaturating conditions. However, our ability to obtain good computer simulations (Figure 1), a 1/T-weighted intensity maximum (Figure 3), and the $4 \mathrm{~K}$ Orme- Johnson plot (Figure 4) allstrongly suggest that, at $9 \mathrm{~K}$ and $0.2 \mathrm{~mW}$, saturation is negligible. 
NOT THE PUBLISHED VERSION; this is the author's final, peer-reviewed manuscript. The published version may be accessed by following the link in the citation at the bottom of the page.

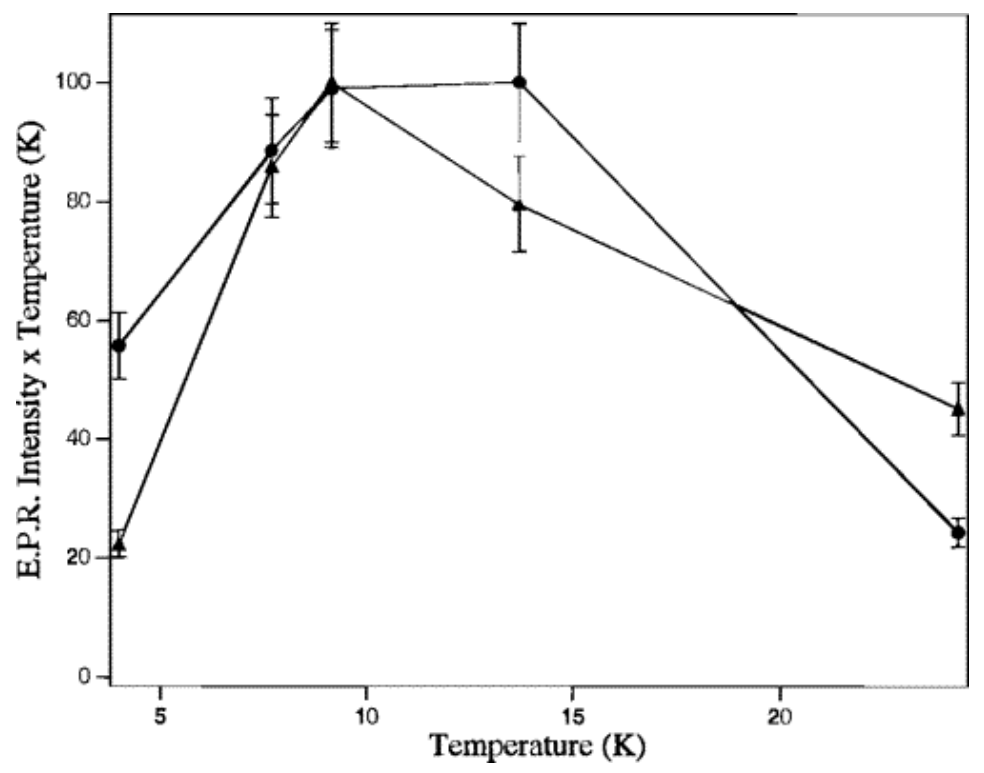

Figure 3 Temperature dependence of the intensity of the EPR signal from [Co_(AAP)]. The plots shows the temperature-weighted EPR intensity of the whole signal (solid triangles) and the hyperfine lines (solid circles) recorded at $0.2 \mathrm{~mW}$ microwave power over a range of temperatures.

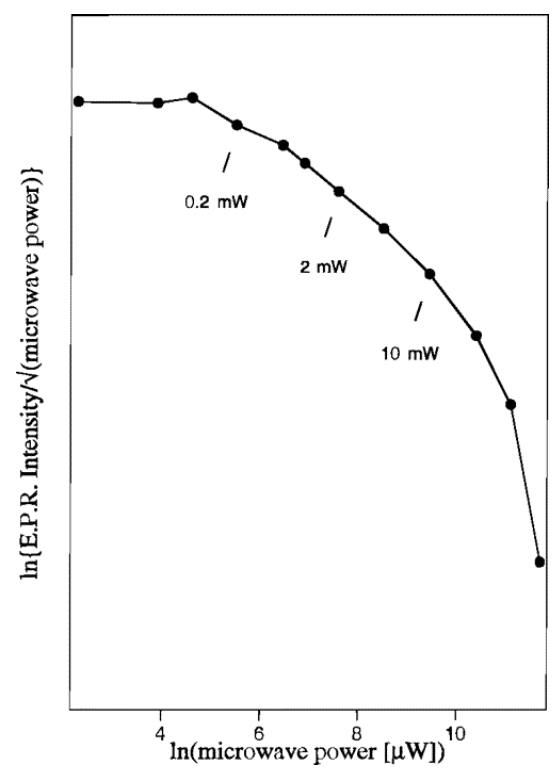

Figure 4 Orme-Johnson plot of the microwave power dependence of the EPR signal from [Co_(AAP)]. The data were collected at $4 \mathrm{~K}$.

As well as causing a loss of signal intensity, another consequence of saturation is gross distortion of the EPR signal. As can clearly be seen from the spectrum recorded at $4 \mathrm{~K}$ and $2 \mathrm{~mW}$ microwave power (Figure 5A), the area above the baseline is much larger than that below the baseline, suggesting that the signal

Journal of the American Chemical Society, Vol 119, No. 8 (February 26, 1997): pg. 1923-1933. DOI. This article is (C) American Chemical Society and permission has been granted for this version to appear in e-Publications@Marquette. American Chemical Society does not grant permission for this article to be further copied/distributed or hosted elsewhere without the express permission from American Chemical Society. 
saturates in an anisotropic manner. Some features have shifted and the hyperfine pattern is barely resolvable. The signal could be simulated (Figure 5B), assuming $g_{\text {eff }}$ values of $5.26,4.25,2.66$ and $6.83,3.28,2.66$ for the two contributing species, although not satisfactorily. These parameters differ from those obtained under the essentially nonsaturating conditions as described above. The closest theoretically allowed values of $5.28,4.28,2.66$ (for $M_{\mathrm{s}}=| \pm 1 / 2\rangle, g_{\text {real }}$ $=2.4, E / D=0.07)$ and $6.83,3.20,3.17\left(\right.$ for $M_{\mathrm{s}}=| \pm 1 / 2\rangle, g_{\text {real }}=2.6$, $E / D=0.25$ ) provide a relatively poor fit (Figure $5 \mathrm{C}$ ) and indicate the extent of distortion of the spectrum. Nevertheless, these simulations yield surprisingly similar values of $g_{\text {real }}$ and $E / D$ to those obtained from the simulations of spectra recorded under essentially nonsaturating conditions. These data suggest that the calculated $g_{\text {real }}$ and $E / D$ values derived from simulations of spectra under saturating conditions vary little from those obtained from nonsaturated spectra. Therefore, while it cannot be unambiguously demonstrated that a microwave power of $0.2 \mathrm{~mW}$ at $9 \mathrm{~K}$ provides absolutely nonsaturating conditions, the parameters derived from simulations of spectra recorded under these conditions appear to be valid. This statement is supported by the quality of the simulations of these spectra and by the excellent agreement of the $g_{\text {eff }}$ values obtained by line-shape simulations and from the Rhombogram program.

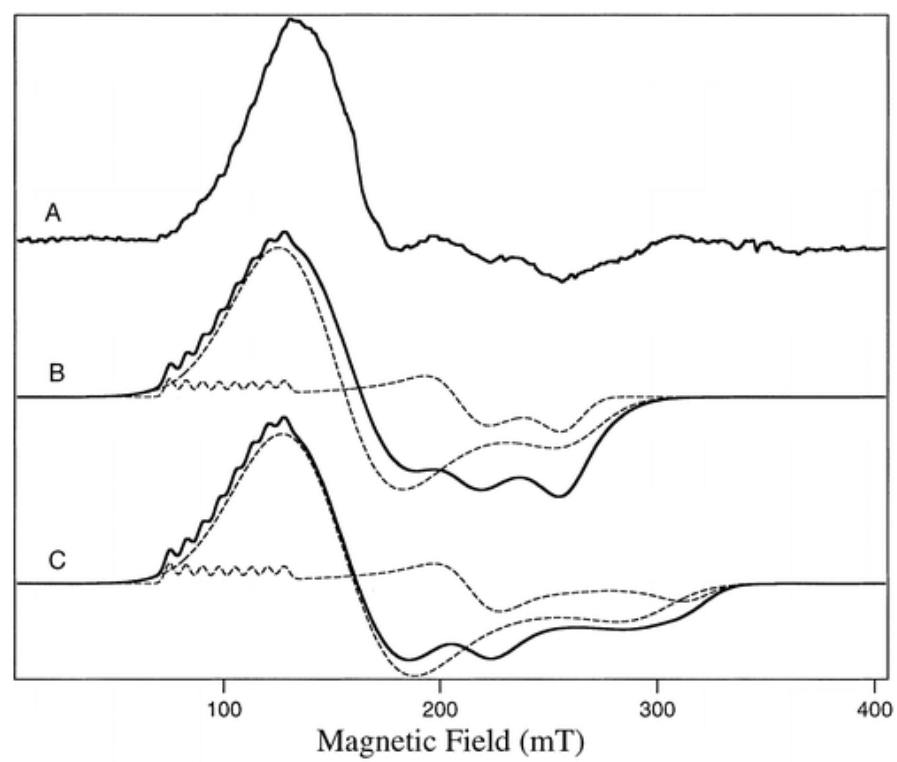

Figure 5 EPR spectrum of [Co-(AAP)] recorded under saturating conditions. Spectrum $A$ was recorded at $4 \mathrm{~K}, 2 \mathrm{~mW}$ microwave power. Simulation of $A(B)$ was the closest fit to the line shape assuming two species [(i) $g=5.26,4.25,2.66$; (ii) $g=6.83,3.28$, 
2.66, $\left.\left.A_{z}=7.2 \mathrm{mT}\right)\right]$. Simulation $\mathrm{C}$ was generated using the closest theoretically allowed parameters of (i) $g=5.28,4.28,2.66, g_{\text {real }}=2.4, E / D=0.07$; (ii) $g=6.83,3.20$, $3.17, A_{z}=7.2 \mathrm{mT}, g_{\text {real }}=2.6, E / D=0.25$.

The gross distortion of the EPR spectrum of [Co-(AAP)] observed under saturating conditions prompts a re-examination of data in the literature. To our knowledge, the effect of saturation on the line shapes of Co(II) EPR spectra or on simulation-derived spectral parameters has not been investigated in any detail, and very few reports mention that care must be taken to ascertain nonsaturating conditions. From Figure 5 it can clearly be seen that the EPR spectrum of [Co-(AAP)] does not saturate in an isotropic fashion. This finding is important because the lack of high field features in $S=3 / 2$ high-spin Co(II) EPR spectra is often taken as being indicative of a spin system with very little rhombic distortion $(E / D \rightarrow 0)$ exhibiting transitions in the $M_{s}=| \pm 3 / 2\rangle$ doublet. In such cases, $g_{x, y}$ approaches zero as does the transition probabilities for these resonances. However, Figure $5 \mathrm{~A}$ clearly shows that under saturating conditions the higher field resonances of an $M_{s}=\left| \pm^{1} / 2\right\rangle$ doublet can lose intensity much more readily than the low field $g_{z}$ resonance. Thus the spectrum may appear to exhibit only one resonance at $g=6$ to 7 and be mistakenly assigned to an $M_{s}=\left| \pm^{3} / 2\right\rangle$ system.

EPR titration of apo-AAP with Co(II) indicated that the observed signal increased in intensity linearly with added $\mathrm{Co}$ (II) until 1 equiv had been added (Figure 6). Further addition of $\mathrm{Co}$ (II) resulted in a linear decrease in intensity and was accompanied by loss of the hyperfine pattern. Beyond 2 equiv, the EPR intensity again began to rise due to free-Co(II). An EPR spectrum of AAP plus 2 equiv of $\mathrm{Co}$ (II) ([CoCo(AAP)]) is shown in Figure 7 along with a computer simulation. The parameters obtained by simulation were $g_{\text {eff( }(1,2,3)}=5.10(5.10)$, 3.85 (3.84), $2.19(2.19) ; M_{\mathrm{s}}=\left| \pm \frac{1}{2}\right\rangle ; g_{\text {real }}=2.25 ; E / D=0.095$. These data are consistent with the second Co(II) ion in AAP occupying an octahedral coordination geometry. The intensity of the observed signal for [CoCo(AAP)] corresponded to $0.13 \mathrm{spin} / \mathrm{mol}$ of $\mathrm{Co}$ (II) added. Some hyperfine structure due to a trace amount of a second species is barely visible on the low-field feature, and computer resolution enhancement techniques enabled measurement of $g_{z}=6.83$ and $A_{z}=$ $7.2 \mathrm{mT}$, similar to [Co-(AAP)]. The small contribution of the hyperfinecontaining species did not warrant its inclusion in the simulation and is 
likely due to trace amounts of [Co-(AAP)]. The low intensity of the EPR signal per mole of $\mathrm{Co}(\mathrm{II})$ in $[\mathrm{CoCo}(\mathrm{AAP})]$ strongly suggest that the two $\mathrm{Co}$ (II) ions in the active site of AAP experience significant spin-spin interaction and are either antiferromagnetically or ferromagnetically coupled. No zero-field splitting lines at higher or lower fields could be detected, suggesting that $J \geq 0.3 \mathrm{~cm}^{-1}$. The signal displayed similar saturation behavior to that of [Co-(AAP)] except that a clear region of $1 / T$ intensity dependence was evident from 12 to $20 \mathrm{~K}$ at microwave powers of $0.2 \mathrm{~mW}$ (Figure 8). As can be seen from Figure 8, this signal also distorts anisotropically; the higher field features are more susceptible to saturation whereas the lower field feature is more readily broadened by relaxation effects at higher temperatures. Since the EPR signal observed from [CoCo(AAP)] is observable only over a very narrow range of temperatures, it is impossible to determine the relaxation regime (i.e., Orbach relaxation). ${ }^{41}$ Therefore, the sign of the zero-field splitting could not be determined.

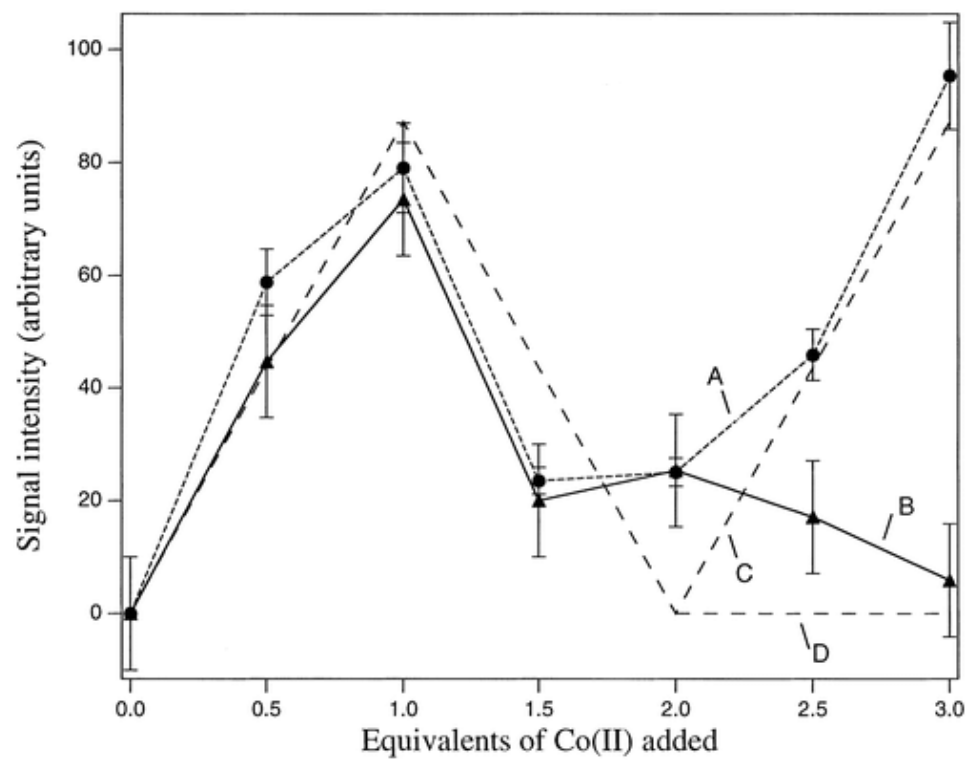

Figure 6 Titration of apo-AAP with Co(II). The integrated intensity of the EPR signal from AAP, plotted as a function of added Co(II), is shown as A. The signal observed upon addition of more than 2 equiv of $\mathrm{Co}$ (II) was due to the contribution of free Co(II). B shows the intensity of the hyperfine lines of the spectra upon addition of $\mathrm{Co}$ (II) to AAP. $\mathrm{C}$ is the expected intensity variation obtained if the two Co(II) ions in [CoCo(AAP)] are assumed to be strongly spin-coupled. $D$ is the expected variation in the intensity of the hyperfine lines, also assuming strong spin-coupling. 
NOT THE PUBLISHED VERSION; this is the author's final, peer-reviewed manuscript. The published version may be accessed by following the link in the citation at the bottom of the page.

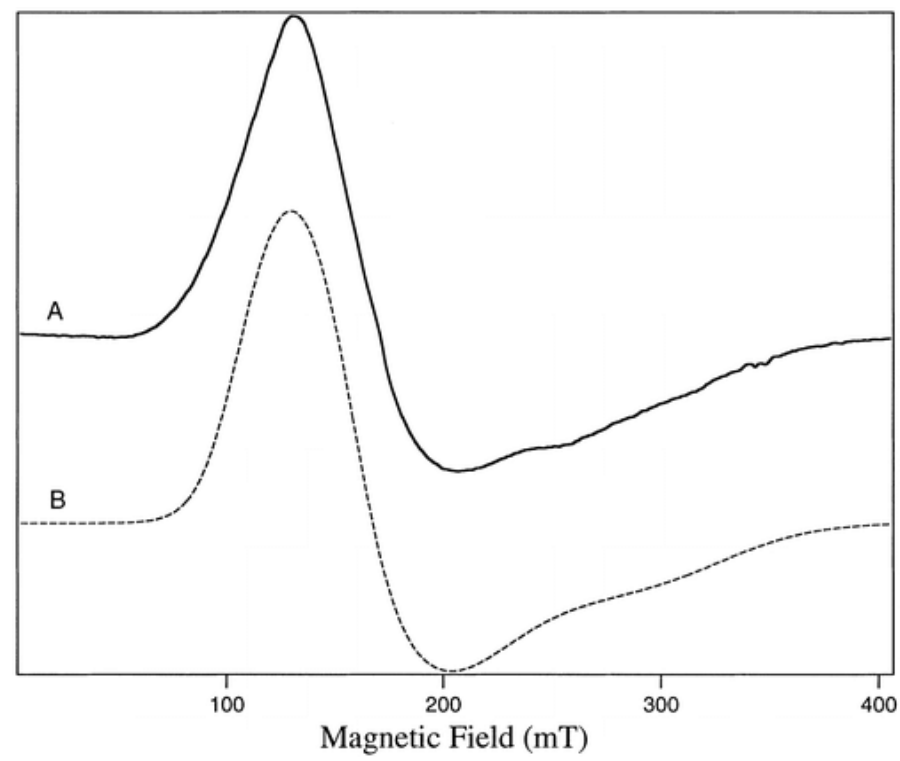

Figure $7(A)$ EPR spectrum of [CoCo(AAP)] at $10 \mathrm{~K}, 0.2 \mathrm{~mW}$ microwave power. Signal A was simulated (B) using the parameters $g_{\text {eff }}=5.10,3.85,2.19$. The signal corresponds to 0.13 spin $\mathrm{mol}^{-1} \mathrm{Co}(\mathrm{II})$.

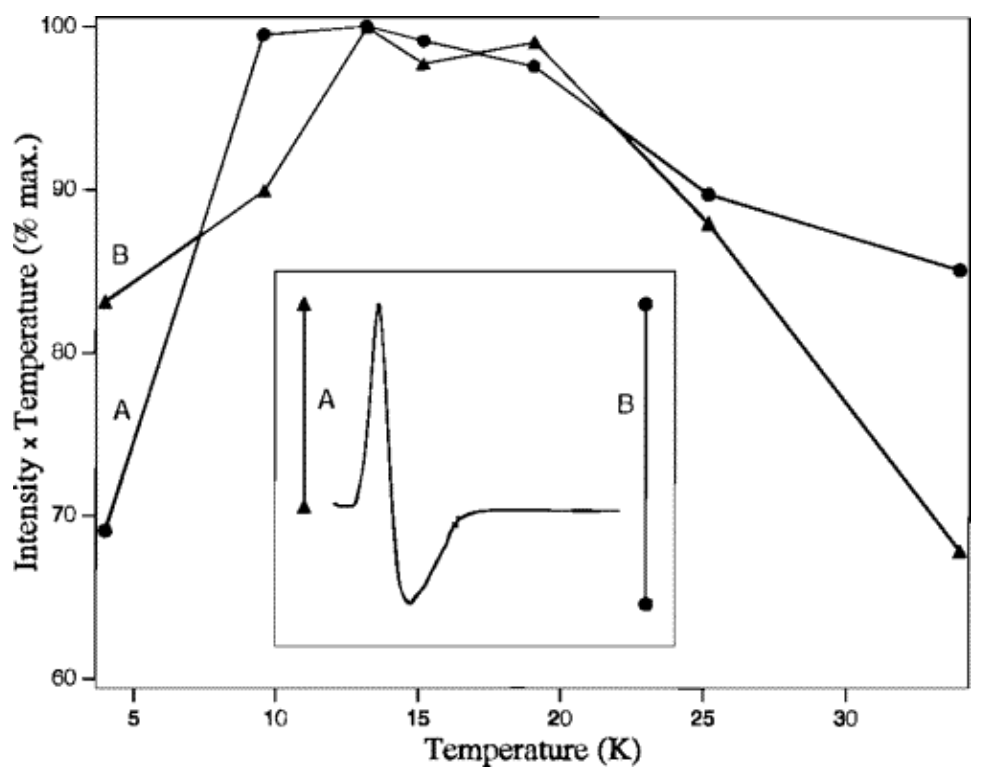

Figure 8 The temperature-weighted intensity of the EPR signal of [CoCo(AAP)] is shown as a function of temperature. Insert: features of the EPR spectrum that were measured.

The $\mathrm{pH}$ dependence of the observed [CoCo(AAP)] EPR signal was investigated by recording spectra at $\mathrm{pH}$ values of 5.6 and 10.0 . The EPR characteristics of the enzyme at $\mathrm{pH} 10$ were indistinguishable from those at $\mathrm{pH} 7.5$ except that no hyperfine structure was visible. However, at pH 5.6 the spectrum was markedly different (Figure 9). At

Journal of the American Chemical Society, Vol 119, No. 8 (February 26, 1997): pg. 1923-1933. DOI. This article is (C) American Chemical Society and permission has been granted for this version to appear in e-Publications@Marquette. American Chemical Society does not grant permission for this article to be further copied/distributed or hosted elsewhere without the express permission from American Chemical Society. 
$4 \mathrm{~K}$ and $0.2 \mathrm{~mW}$ microwave power, the spectrum appears to consist of a single species and can be reasonably simulated $\left(g_{\text {eff( }(1,2,3)}=5.62\right.$ (5.58), $3.32(3.30), 1.94(2.07) ; M_{s}=\left| \pm^{1} / 2\right\rangle ; g_{\text {real }}=2.27 ; E / D=$ 0.18 ) (Figure $9, A$ and $B$ ). Therefore, the $g$ value for the low $\mathrm{pH}$ species is very similar to that obtained at $\mathrm{pH}$ 7.5. At higher temperatures, however, hyperfine structure becomes visible and is optimally resolved at 7-12 K. Simulation of the observed hyperfine signals (Figure 9D) revealed that $g_{z}=6.83$ and $A_{z}=7.2 \mathrm{mT}$. The other parameters used for the simulation were identical with those for the hyperfine-structure-containing species of [Co-(AAP)]. These data are also consistent with the presence of a bridging water molecule that is in equilibrium with a bridging hydroxide.

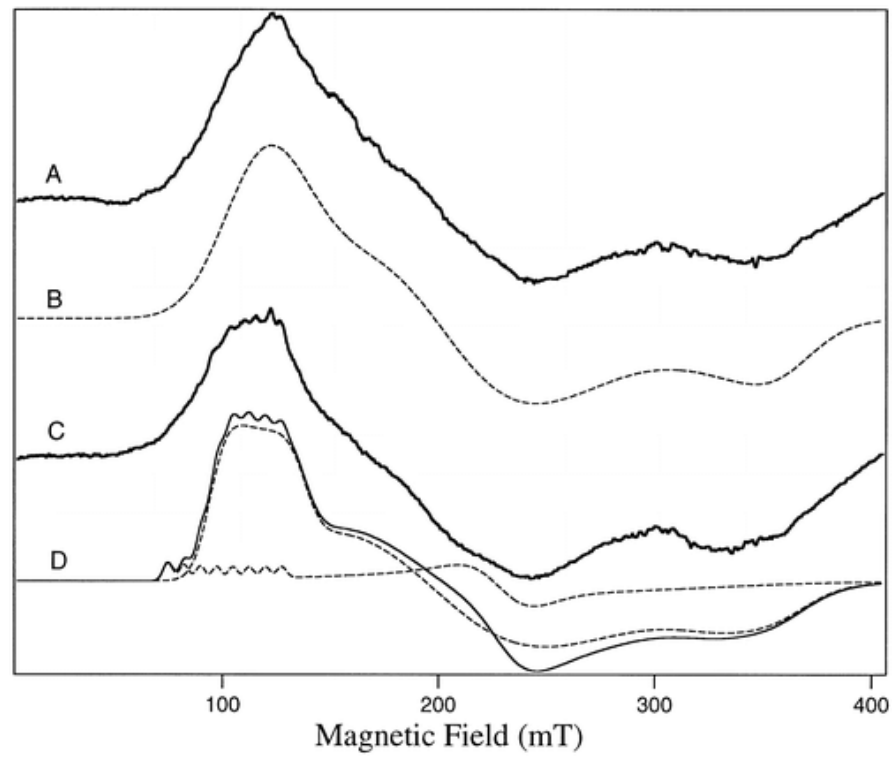

Figure 9 The low-pH EPR spectrum of [CoCo(AAP)]. Spectra $A$ and $C$ are of the same [CoCo(AAP)] sample in $50 \mathrm{mM}$ Mes. buffer, $\mathrm{pH}$ 5.6. Spectrum $A$ was recorded at $4 \mathrm{~K}$, $0.2 \mathrm{~mW}$ microwave power. Spectrum $\mathrm{C}$ was recorded at $10 \mathrm{~K}, 0.2 \mathrm{~mW}$. B is a simulation using $g_{\text {eff }}=5.62,3.32,1.94$. D is a simulation assuming two species with parameters (i) $g_{\text {eff }}=5.62,3.32,1.94$; (ii) $g_{\text {eff }}=6.83,2.95,2.05, A_{z}=7.2 \mathrm{mT}$.

EPR Characterization of an $\mathbf{S}=\mathbf{3}$ Species. Until recently, the applicability of EPR spectroscopy to biologically relevant species was considered to be limited to Kramers systems of spin $S=(n+1 / 2)$ where $n$ is an integer or zero. However, unusual EPR resonances observed in spectra obtained from cytochrome $c$ oxidase were suggested to be due to an integral spin system. This led Hagen ${ }^{64}$ to formalize a methodology with which to study integral spin systems using parallel mode EPR. In contrast to orthodox (perpendicular mode) 
EPR, with parallel mode EPR the magnetic vector of the standing microwave is aligned parallel to the applied magnetic field. The advantage of parallel mode EPR to study integer spin systems arises because zero-field splittings are often sufficiently large such that the relatively small Zeeman interaction does not converge the energy levels. Thus, the $\Delta m_{\mathrm{s}}= \pm 1$ transition may not be accessible. In parallel mode, the selection rule becomes $\Delta m_{\mathrm{s}}=0$ and transitions between higher non-Kramers doublets become observable in cases where part of the zero-field splitting distribution envelope falls within the accessible range of energies and where the applied field has a nonzero projection on the molecular z-axis. For transition metal ions, the levels of the non-Kramers doublet responsible for observed parallel mode EPR spectra diverge rapidly with applied magnetic field. Thus, the spectral features are usually observed at low field. The selectivity of parallel mode EPR for integral spin systems is useful when overlapping spectra due to $S=(n+1 / 2)$ species preclude observation of an integer spin system when using perpendicular mode EPR. This is particularly true for systems with significant perpendicular mode EPR absorption at low field, such as high-spin Co(II).

Perpendicular mode EPR titration of apo-AAP with Co(II) revealed a low-field signal extending out of zero field in samples with more than 1 equiv of Co(II) added (Figure 10B). This type of EPR absorption is indicative of an integral spin system. The observed lowfield feature was quantified by measuring the difference between the first derivative of the EPR absorption at $5 \mathrm{mT}$ and $45 \mathrm{mT}$ applied magnetic field. The change in intensity of this feature as $\mathrm{Co}$ (II) was added to apo-AAP is plotted in Figure 11. The low-field feature was not present in samples to which less than 1 equiv of $\mathrm{Co}$ (II) had been added. As more Co(II) was added, the intensity of this feature was found to increase linearly between 1 and 2 equiv of $\mathrm{Co}$ (II). No further increase in the intensity of the low-field feature was observed upon addition of $\mathrm{Co}$ (II) beyond 2 equiv. These data provide the first definitive evidence that metal binding to AAP occurs sequentially since no exchange-coupling is observed until more than 1 equiv of $\mathrm{Co}$ (II) has been added. 
NOT THE PUBLISHED VERSION; this is the author's final, peer-reviewed manuscript. The published version may be accessed by following the link in the citation at the bottom of the page.

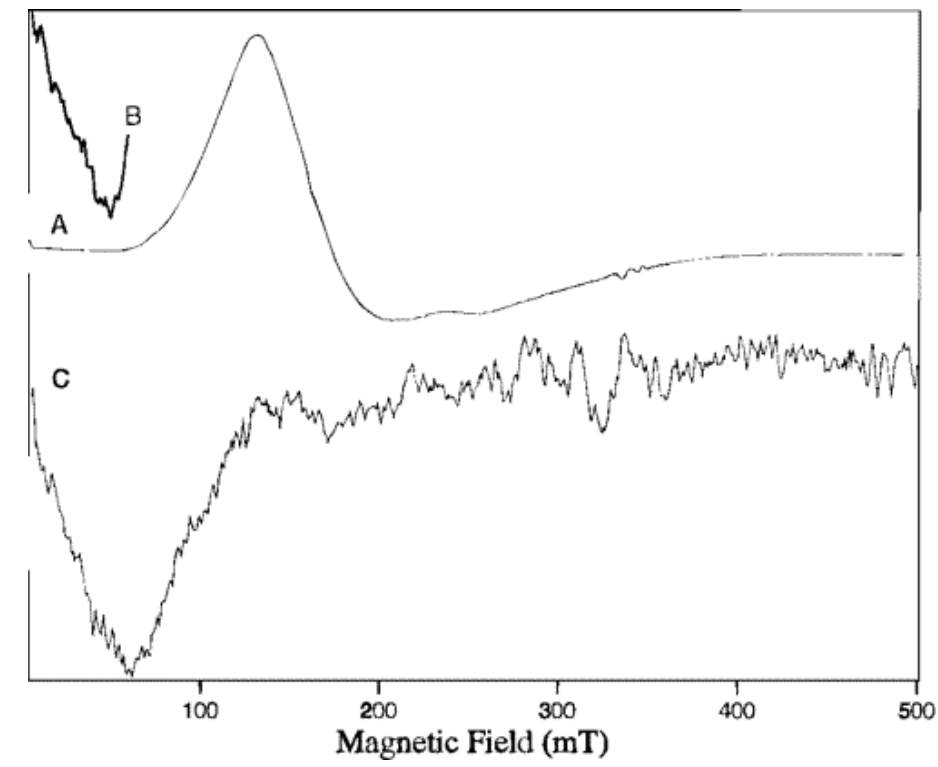

Figure $10 S=3 / 2$ and $S=3$ EPR signals from [CoCo(AAP)]. Spectrum $A$ is the perpendicular mode EPR signal from a sample of AAP containing 1.5 equiv of $\mathrm{Co}$ (II). Spectrum $A$ was recorded at $10 \mathrm{~K}, 0.2 \mathrm{~mW}$ microwave power. Inset $B$ : the low-field region of spectrum A plotted with a 32 -fold increase in signal intensity. Spectrum $C$ is the parallel mode EPR signal of the same sample recorded at $4.2 \mathrm{~K}, 5 \mathrm{~mW}$ microwave power.

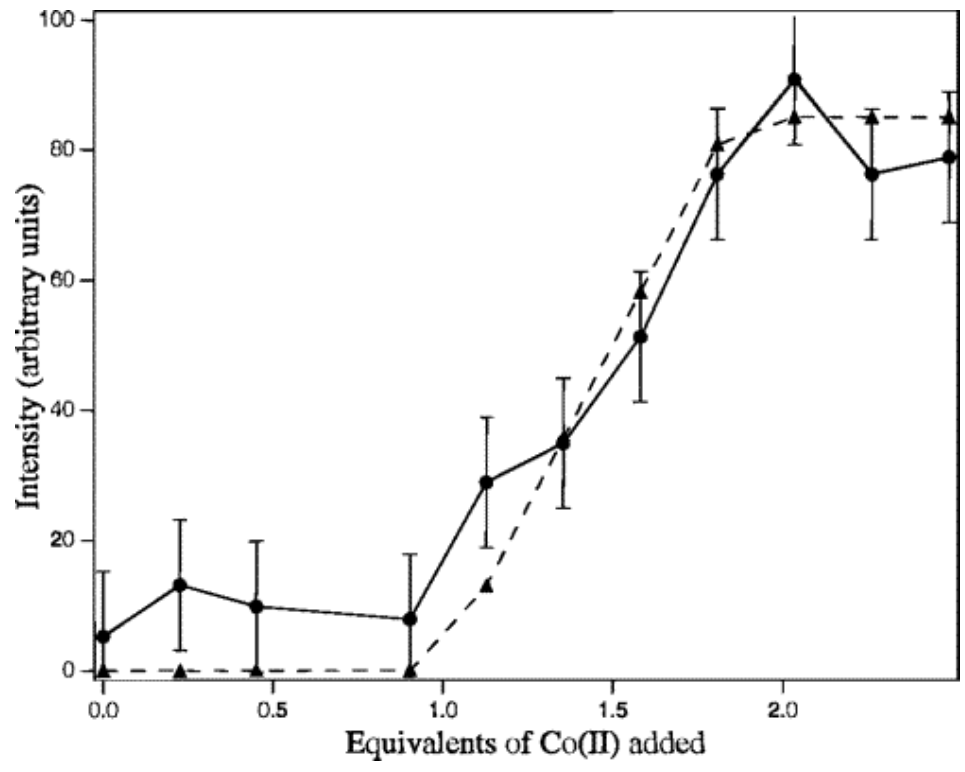

Figure 11 Titration of apo-AAP with Co(II) followed by the intensity of the low-field EPR feature corresponding to $B$ in Figure 10. The solid line is a plot of the experimental data and the error bars apply to this line. The dashed line corresponds to intensities calculated assuming that the species responsible for the feature is a dicobalt(II) species. 
Coincident with the appearance of the low-field perpendicular mode signal was the appearance of a parallel mode EPR signal, as shown in Figure 10C, with $g \sim 12$. These data are indicative of the presence of an $S=3$ spin system that is related to the presence of two Co(II) ions per enzyme molecule. An $S=3$ spin system would arise from ferromagnetic coupling of two high-spin $S=3 / 2 \mathrm{Co}$ (II) ions in the enzyme active site. Thus, the magnetic orbitals of the two Co(II) ions in AAP must be orthogonal to one another. As is evident from Figure 10, the low-field perpendicular mode EPR signal was obliterated at field strengths greater than $50 \mathrm{mT}$ by the $S=3 / 2$ signal; however, in parallel mode the only signal observable was the low-field signal at $g \sim 12$. The position of this resonance varied with the microwave power suggesting that it is readily saturated. At the lowest microwave power at which we could detect a signal with a reasonable signal to noise ratio $(0.2 \mathrm{~mW})$, the minimum position of the signal corresponded to $g=12.8$. At higher microwave powers slightly lower $g_{\text {eff }}$ values were observed (for example, $g_{\text {eff }}=11.8$ at $0.5 \mathrm{~mW}$; see Figure $10 \mathrm{C}$ ) which is typical for integer spin signals. In addition, this signal was observed only at temperatures below $12 \mathrm{~K}$ and thus appears to be due to a ground-state transition. The lack of an $S=3$ signal in [Co-(AAP)], [CoZn(AAP)], and [ZnCo(AAP)] confirms that the two $\mathrm{Co}$ (II) ions in AAP are ferromagnetically coupled. The only other reported EPR spectrum of a putatively spin-coupled dicobalt(II) system was observed in the low-field region of the perpendicular mode spectrum of Co(II) substituted hemocyanin; ${ }^{42}$ however, no data were provided to confirm this signal.

Since 1982, the theory and methodology of EPR integral spin systems have advanced considerably ${ }^{65,66}$ and a number of metalloprotein systems have been studied. These include the heme-copper centers in cytochrome oxidases, ${ }^{67-69}$ the $S=2$ states of the [3Fe-4S] cluster from Thermus thermophilus ferredoxin, ${ }^{70}$ and the [3Fe-4S] cluster of Escherichia coli aconitase-A, ${ }^{55}$ as well as the dinuclear iron center of $E$. coli ribonucleotide reductase. ${ }^{71} S=2$ signals have also been detected from the two mononuclear Fe(II) centers in reduced desulfoferredoxin from Desulfovibrio gigas. ${ }^{72}$ In addition, higher spin systems have been successfully probed including the $S=3$ $\mathrm{P}$ clusters of the nitrogenase MoFe protein from Azotobacter vinelandii ${ }^{73}$ and other organisms, ${ }^{74}$ and the $S=4$ center of the 
prismane protein from D. gigas. ${ }^{75}$ However, obtaining zero-field splitting parameters from parallel mode spectra is nontrivial. Unlike perpendicular mode EPR spectra, simulations of parallel mode signals depend critically upon the intensity of the signal. The intensity of the [CoCo(AAP)] $S=3$ signal is dependent upon both the concentration of the spin system and of the proportion of the zero-field splitting energy distribution envelope that is accessible to the X-band microwave. ${ }^{64-66}$ Thus, meaningful zero-field splittings can only be obtained where the spin concentration is accurately known. The existence of an $S=3 / 2$ perpendicular mode signal in [ $\mathrm{CoCo}(\mathrm{AAP})]$ samples that also exhibit an $S=3$ parallel mode signal is indicative of a population distribution containing a mixture of $S=3 / 2, S=3$, and possibly $S=0$ species. The presence of mixed magnetic species in $\mathrm{Co}$ (II) substituted proteins is not uncommon and in some cases can be related to the sensitivity of the EPR parameters to angular co-orientational microheterogeneities analogous to the structural microheterogeneities responsible for $g$ strain of mono-Co(II) ions in proteins. ${ }^{32,39,42,43}$

The line width of the parallel mode EPR signal at full width at half-maximum is $\sim 70 \mathrm{mT}$. One possible explanation for this signal being rather broad is that it is split by the hyperfine interaction due to the two ${ }^{59} \mathrm{Co}$ nuclei. No resolvable hyperfine structure could be detected in the present study; however, resolvable hyperfine structure would be highly unlikely since coupling of the electronic spin to two ${ }^{59} \mathrm{Co}$ nuclei would result in up to 64 lines. While hyperfine structure is clearly resolvable in the perpendicular mode spectrum of [Co-(AAP)], it is not in [CoCo(AAP)]. Moreover, coupling of the electronic spin to only a single ${ }^{59} \mathrm{Co}$ nucleus with a coupling constant of $7.5 \mathrm{mT}$, the value found for $A_{z}$ in the perpendicular mode spectrum of [Co-(AAP)], would introduce a broadening of $60 \mathrm{mT}$. Examination of parallel and perpendicular mode $S=3$ spectra in the literature, particularly that of the $\mathrm{P}$-clusters of $A$. vinelandii nitrogenase MoFe protein, ${ }^{73}$ suggest that the line widths of $S=3$ spectra recorded in both modes are comparable. For [CoCo(AAP)], the line width of the parallel mode signal is similar to that of the perpendicular mode signal. The comparable line widths suggest that the hyperfine coupling in the parallel mode species is of a similar order to that in the perpendicular mode species. An additional contribution to the line width may arise from $E / D$ strain. That significant $g$ and $A$ strain needs to be invoked to 
simulate the intensity distribution of the ${ }^{59} \mathrm{Co}$ hyperfine lines in the perpendicular mode spectrum of [Co-(AAP)] is indicative of the Co(II) ions experiencing a microheterogenous distribution of ligand fields (i.e., E/D strain).

The parallel mode EPR signal due to [CoCo(AAP)] was found to be dependent on both $\mathrm{pH}$ and added oxidant. As the $\mathrm{pH}$ was increased from 5.9 to 10.0 , the intensity of the $S=3$ parallel mode EPR signal decreased. The enzymatic activity of these samples assayed at $\mathrm{pH} 8.0$ remained constant and no perpendicular mode EPR signal of free Co(II) could be detected. Concomitant with the loss of the $S=3$ signal, an increase in the $S=3 / 2$ signal due to [CoCo(AAP)] was observed. Thus enzyme degradation or metal loss does not occur. These data suggest that the $S=3$ species giving rise to the parallel mode signal is dependent upon an ionizable group. This is consistent with recent kinetic and X-ray crystallographic studies that indicate at $\mathrm{pH}$ values less than 8.0 , the $\mathrm{pH}$ for maximal activity, the dimetal cluster contains a ( $\mu$-aqua)( $\mu$-carboxylato) core. Thus at high $\mathrm{pH}$ values, the bridging water molecule likely becomes deprotonated and the spin-coupling between the two Co(II) ions is altered. These data suggest that the dicobalt(II) AAP enzyme also contains a ( $\mu$-aqua)( $\mu$ carboxylato) core and that the bridging water molecule mediates the ferromagnetic coupling between the two $\mathrm{Co}$ (II) ions at $\mathrm{pH}$ 7.5. This deprotonation likely causes the spin-population distribution observed for $[\mathrm{CoCo}(\mathrm{AAP})]$.

The observed parallel mode signal was less intense than the perpendicular mode $S=3 / 2$ signals, and, therefore, we sought to eliminate the possibility that the signal could be due to adventitious $\mathrm{Fe}$ (II). ${ }^{64}$ Figure 12 shows the effect of hydrogen peroxide on the EPR spectrum of [CoCo(AAP)]. Ten equivalents of hydrogen peroxide was rapidly mixed with [CoCo(AAP)], incubated for $5 \mathrm{~s}$, and then rapidly frozen. The parallel mode signal was completely abolished, and the broad, featureless perpendicular mode EPR signal, characteristic of $[\mathrm{CoCo}(\mathrm{AAP})]$, was replaced by a signal that exhibited clear ${ }^{59} \mathrm{Co}$ hyperfine structure. Similar spectra were observed for [Co-(AAP)], the heterometallic [CoZn(AAP)] complex, as well as an air oxidized [CoCo(AAP)] sample (data not shown). Particularly important is the lack of an intense $g=4.3$ resonance in the perpendicular mode 
spectrum that would indicate the presence of Fe(III). These data confirm that the observed $g \sim 12$ signal is not due to an Fe(II) impurity.

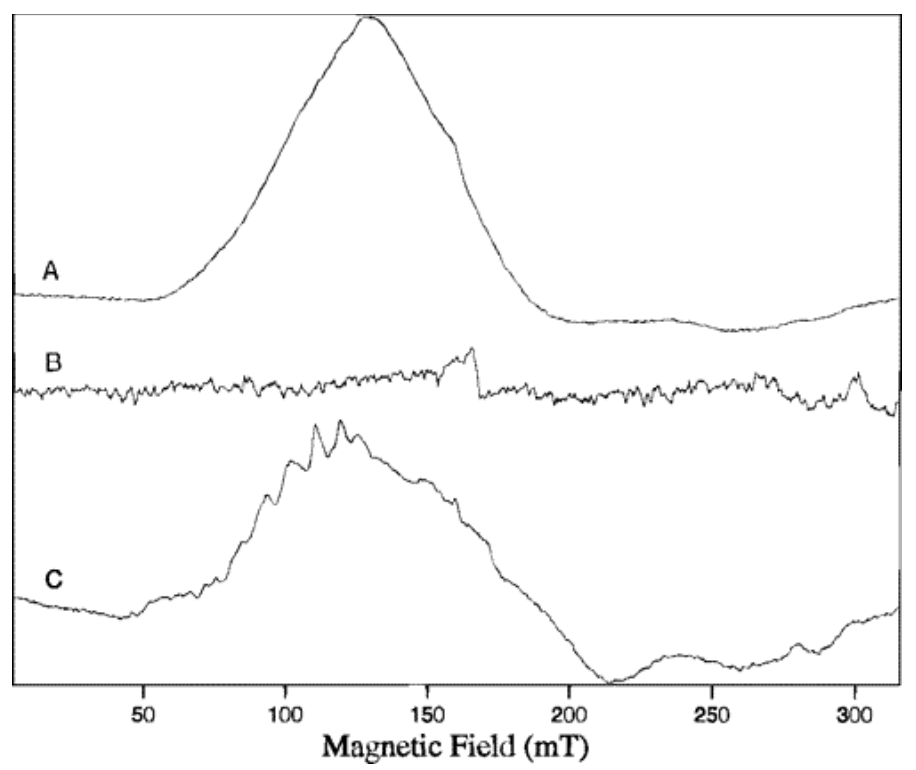

Figure 12 The effect of hydrogen peroxide on the EPR spectrum of [CoCo(AAP)]. Spectrum $A$ is the perpendicular mode EPR signal of [CoCo(AAP)] recorded at $5 \mathrm{~K}, 2$ $\mathrm{mW}$ microwave power. The sample also exhibited a parallel mode EPR signal similar to that shown in Figure 10C. The sample was treated with 10 equiv of hydrogen peroxide for $5 \mathrm{~s}$ and rapidly frozen. The parallel (B) and perpendicular mode (C) EPR spectra were then recorded at $5 \mathrm{~K}, 2 \mathrm{~mW}$ microwave power.

In order to probe the mechanism of peptide hydrolysis by AAP, we studied the effect of the competitive inhibitor 1-butaneboronic acid (BuBA) on the $S=3$ EPR signal of [CoCo(AAP)]. The addition of 1 equiv of BuBA to [CoCo(AAP)] resulted in the immediate loss of the $S$ $=3$ EPR signal. These data suggest that, upon BuBA binding, the $\mu-$ aquo bridge between the $\mathrm{Co}$ (II) ions is lost. Since AAP is ca. $80 \%$ active with only a single $\mathrm{Zn}$ (II) ion bound and recent fluoride inhibition studies indicate that fluoride binding occurs only after substrate binding, ${ }^{76}$ it seems likely that the bridging water molecule becomes terminal upon substrate binding and is bound to the catalytic metal site. Thus, the exchange-coupling between the two Co(II) ions is lost and the terminal water/hydroxyl likely represents the hydroxylating agent in the enzymatic reaction.

\section{Proposed Mechanism of Peptide Hydrolysis by AAP.} Combination of the available X-ray crystallographic and kinetic data 
with the present spectroscopic data allows a detailed catalytic mechanism to be proposed for AAP (Figure 13). In the resting [CoCo(AAP)] enzyme, the presence of the $S=3$ spin system clearly demonstrates that the metal ions communicate electronically with each other and, therefore, are capable of modulating each other's catalytic properties. Based on the absorption data reported for [Co-(AAP)], $[\mathrm{CoCo}(A A P)]$, and $[\mathrm{ZnCo}(A A P)]$ in the presence and absence of BuBA, it was proposed that BuBA binds to the first metal center but not the second. These data are consistent with the loss of the $S=3$ EPR signal of $[\mathrm{CoCo}(\mathrm{AAP})]$ upon BuBA binding. Based on X-ray crystallographic data of a transition-state analogue inhibitor bound AAP complex, the carbonyl oxygen of the peptide indeed binds directly to the catalytic metal ion. This binding scheme is consistent with the large negative entropy and large positive enthalpy of activation reported for AAP. ${ }^{47}$ Thus, the bridging water/hydroxide becomes terminal and appears to represent the hydroxylating agent in the enzymatic reaction. We therefore propose that only the first metal ion is directly involved in catalysis and that the second metal ion stabilizes the transition state of the hydrolytic reaction. This point is emphasized by the fact that the addition of 1 mole of $\mathrm{Cu}$ (II) or $\mathrm{Ni}$ (II) to apo-AAP followed by the addition of $\mathrm{Zn}$ (II) enhances the enzymatic activity nearly 100 -fold. ${ }^{29}$ Thus, the dinuclearity of the active site is intimately involved in catalysis.

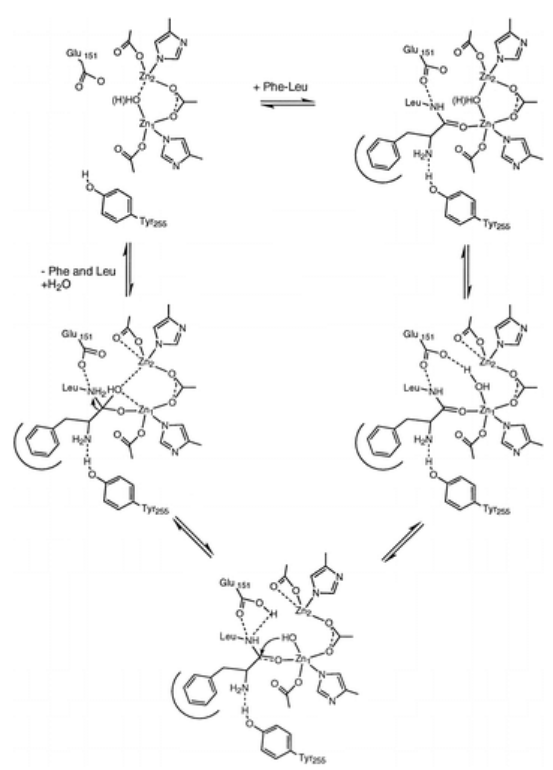

Figure 13 Proposed mechanism for hydrolysis of $\mathrm{N}$-terminal amino acids from polypeptide chains by AAP.

Journal of the American Chemical Society, Vol 119, No. 8 (February 26, 1997): pg. 1923-1933. DOI. This article is (C American Chemical Society and permission has been granted for this version to appear in e-Publications@Marquette. American Chemical Society does not grant permission for this article to be further copied/distributed or hosted elsewhere without the express permission from American Chemical Society. 
Since an active site carboxylate group, Glu151, has been implicated in the catalytic process from X-ray crystallographic data, the catalytic mechanism of AAP may resemble that proposed for thermolysin or carboxypeptidase A. ${ }^{54}$ Similar to that of Glu270 in carboxypeptidase A, the role of Glu151 in catalysis is likely to assist in deprotonation of the terminal water molecule to the nucleophilic hydroxo moiety. ${ }^{77}$ Thus, the metal-bound hydroxide can attack the activated scissile carbonyl carbon of the peptide substrate forming a gem-diolate intermediate complex that is stabilized by coordination of both oxygen atoms to the dimetal(II) center. Glu151 can then provide an additional proton to the penultimate amino nitrogen returning it to its ionized state. The $\mathrm{C}-\mathrm{N}$ bond-breaking step would be the ratelimiting step, consistent with recent thermodynamic studies that indicate that product release is the slow step at $25^{\circ} \mathrm{C} .{ }^{47}$ Finally, the dinuclear metal cluster releases the cleaved peptides and adds a water molecule that bridges between the two metal ions.

\section{Conclusion}

The present study has shown that EPR simulation of a single $S=$ $3 / 2$ high-spin Co(II) species can be carried out by treating the system as an effective $S=1 / 2$ system. Detailed interpretation of high-spin Co(II) EPR spectra has proved to be a major obstacle when using $\mathrm{Co}$ (II) as a probe to study protein structure-function relationships. Our simulations show that $g_{\text {real }}$ and $E / D$ values can be extracted from the $g_{\text {eff }}$ values obtained by simulation since the observed resonance positions, $g_{\operatorname{eff}(x, y, z)}$, are largely dictated by the zero-field splitting. Our data also suggest that the calculated $g_{\text {real }}$ and $E / D$ values derived from simulations of spectra under slightly saturating conditions will vary little from those obtained from nonsaturated spectra. In cases where two species are present, the observed hyperfine pattern due to ${ }^{59} \mathrm{Co}$ is not centered on the low-field absorption feature. Delineating these two species, which arise from $S=1 / 2$ Kramers doublets of the $S=3 / 2$ manifold, allows spectral simulation of each signal. The simulations presented provide values for $g_{\text {real }}, g_{\text {eff, }}$ and $E / D$, the validity of which also provides a means of verifying the line-shape simulations. We have also provided the first definitive evidence of ferromagnetically coupled high-spin $(S=3 / 2) \mathrm{Co}(\mathrm{II})$ ions in a dinuclear cluster. Signals characteristic of an $S=3$ system were observed at $g=12.8$ under 
nonsaturating conditions. The effect of $\mathrm{pH}$, added peroxide, and the coordination of a competitive inhibitor on the signal both confirm the origin of the signal and determine the nature of the interaction between the two Co(II) ions in substituted AAP. Combination of these data with the previously published structural and kinetic results has allowed us to propose a detailed catalytic mechanism for AAP. These data provide new insight into hydrolytic reactions catalyzed by other dinuclear hydrolases and the roles of both metal ions ion catalysis.

\section{Acknowledgment}

The authors wish to acknowledge Professor Michael P. Hendrich for helpful discussions. The program Rhombogram was kindly supplied by Professor Wilfred R. Hagen. This work was supported by the National Science Foundation (CHE-9422098; RCH). The Bruker ESP-300E EPR spectrometer was purchased with funds provided by the National Science Foundation (BIR9413530) and Utah State University.

\section{References}

${ }^{1}$ Sorrell, T. N. Tetrahedron 1989, 45, 3-68.

${ }^{2}$ Karlin, K. D.; Tyeklar, Z. Bioinorganic Chemistry of Copper; Chapman \& Hill: New York, NY, 1993.

${ }^{3}$ Vallee, B. L.; Auld, D. S. Biochemistry 1993, 32, 6493-6500.

${ }^{4}$ Hough, E.; Hansen, L. K.; Birknes, B.; Jynge, K.; Hansen, S.; Horvik, A.; Little, C.; Dodson, E.; Derewenda, Z. Nature 1989, 338, 357-360.

${ }^{5}$ Kim, E. E.; Wyckoff, H. W. J. Mol. Biol. 1991, 218, 449-464.

${ }^{6}$ Bone, R.; Frank, L.; Springer, J. P.; Pollack, S. J.; Osborne, S.-A.; Atack, J. R.; Knowles, M. R.; McAllister, G.; Ragan, C. I.; Broughton, H. B.; Baker, R.; Fletcher, S. R. Biochemistry 1994, 33, 9460-9467.

${ }^{7}$ Beese, L. S.; Steitz, T. A. EMBO J. 1991, 10, 25-33.

${ }^{8}$ Davies, J. F.; Hostomska, Z.; Hostomsky, Z.; Jordan, S. R.; Mathews, D. A. Science 1991, 252, 88-95.

${ }^{9}$ Lahm, A.; Volbeda, A.; Suck, D. J. Mol. Biol. 1990, 215, 207-210.

${ }^{10}$ Jabri, E.; Carr, M. B.; Hausinger, R. P.; Karplus, P. A. Science 1995, 268, 998-1004.

${ }^{11} \mathrm{Holz}$, R. C.; Que, L., Jr.; Ming, L.-J. J. Am. Chem. Soc. 1992, 114, 4434-4436.

${ }^{12}$ True, A. E.; Scarrow, R. C.; Randall, C. R.; Holz, R. C.; Que, L. J. J. Am. Chem. Soc. 1993, 115, 4246-4255.

${ }^{13}$ Sträter, N.; Klabunde, T.; Tucker, P.; Witzel, H.; Krebs, B. Science 1995, 268, 1489-1492. 
NOT THE PUBLISHED VERSION; this is the author's final, peer-reviewed manuscript. The published version may be accessed by following the link in the citation at the bottom of the page.

${ }^{14}$ Roderick, S. L.; Matthews, B. W. Biochemistry 1993, 32, 3907-3912.

${ }^{15}$ Burley, S. K.; David, P. R.; Taylor, A.; Lipscomb, W. N. Proc. Natl. Acad.

Sci. U.S.A. 1990, 87, 6878-6882.

${ }^{16}$ Burley, S. K.; David, P. R.; Sweet, R. M.; Taylor, A.; Lipscomb, W. N. J. Mol. Biol. 1992, 224, 113-140.

${ }^{17}$ Chevrier, B.; Schalk, C.; D'Orchymont, H.; Rondeau, J.-M.; Moras, D.;

Tarnus, C. Structure 1994, 2, 283-291.

${ }^{18}$ Fenton, D. E.; Okawa, H. J. Chem. Soc., Dalton Trans. 1993, 1349-1357.

${ }^{19}$ Feig, A. L. ; Lippard, S. J. Chem. Rev. 1994, 94, 759-805.

${ }^{20}$ Karlin, K. D. Science 1993, 261, 701-708.

${ }^{21}$ Que, L., Jr.; True, A. E. Dinuclear Iron- and Manganese-Oxo Sites in Biology; John Wiley: New York, 1990; Vol. 38, pp 97-200.

${ }^{22}$ Chin, J. Acc. Chem. Res. 1991, 24, 145-152.

${ }^{23}$ Lai, K.; Dave, K. I.; Wild, J. R. J. Biol. Chem. 1994, 269, 16579-16584.

${ }^{24}$ Menger, F. M.; Gan, L. H.; Johnson, E.; Durst, D. H. J. Am. Chem. Soc.

1987, 109, 2800-2803.

${ }^{25}$ Norland, P.; Sjöberg, B.-M.; Eklund, H. Nature 1990, 345, 593-598.

${ }^{26}$ Rosenzweig, A. C.; Federick, C. A.; Lippard, S. J.; Nordlund, P. Nature 1993, 366, 537-543.

${ }^{27}$ deMare, F.; Kurtz, D. M.; Nordland, P. Nature Struct. Bio. 1996, 3, 539-546.

${ }^{28}$ Ben-Bassat, A.; Bauer, K.; Chang, S.-Y.; Myambo, K.; Boosman, A.; Chang, S. J. Bacteriol. 1987, 169, 751-757.

${ }^{29}$ Prescott, J. M.; Wagner, F. W.; Holmquist, B.; Vallee, B. L. Biochem. Biophys. Res. Commun. 1983, 114, 646-652.

30Prescott, J. M.; Wagner, F. W.; Holmquist, B.; Vallee, B. L. Biochemistry 1985, 24, 5350-5356.

${ }^{31}$ Lebioda, L.; Stec, B. J. Am. Chem. Soc. 1989, 111, 8511-8513.

${ }^{32}$ Rose, S. L.; Dickinson, L. C.; Westhead, E. W. J. Biol. Chem. 1984, 259, 4405-4413

${ }^{33}$ Lai, K.; Dave, K. I.; Wild, J. R. J. Biol. Chem. 1994, 269, 16579-16584.

${ }^{34}$ Omburo, G.; Mullins, L. S.; Raushel, F. M. Biochemistry 1993, 32, 9148-9155.

${ }^{35}$ Chae, M. Y.; Omburo, G. A.; Lindahl, P. A.; Raushel, F. M. Arch. Biochem. Biophys. 1995, 316, 765-772.

${ }^{36}$ Bicknell, R.; Hanson, G. R.; Holmquist, B.; Little, C. Biochemistry 1986, 25, 4219-4223.

${ }^{37}$ Elgren, T. E.; Ming, L.-J.; Que, L. Inorg. Chem. 1994, 33, 891-894.

${ }^{38}$ Kuo, J. M.; Raushel, F. M. Biochemistry 1994, 33, 4265-4272.

${ }^{39}$ Martinelli, R. A.; Hanson, G. R.; Thompson, J. S.; Holmquist, B.; Pilbrow, J. R.; Auld, D. S.; Vallee, B. L. Biochemistry 1989, 28, 2251-2258.

${ }^{40}$ Sellin, S.; Eriksson, L. E. G.; Aronsson, A.-C.; Mannervik, B. J. Biol. Chem. 1983, 258, 2091-2093. 
NOT THE PUBLISHED VERSION; this is the author's final, peer-reviewed manuscript. The published version may be accessed by following the link in the citation at the bottom of the page.

${ }^{41}$ Werth, M. T.; Tang, S.-F.; Formicka, G.; Zeppezauer, M.; Johnson, M. K. Inorg. Chem. 1995, 34, 218-228.

${ }^{42}$ Bubacco, L.; Magliozzo, R. S.; Beltramini, M.; Salvato, S.; Peisach, J. Biochemistry 1992, 31, 9294-9303.

${ }^{43}$ Grell, E.; Bray, R. C. Biochim. Biophys. Acta 1971, 236, 503-506.

${ }^{44}$ Bencini, A.; Bertini, I.; Canti, G.; Gatteschi, D.; Luchinat, C. J. Inorg. Biochem. 1981, 14, 81-91.

${ }^{45}$ Makinen, M. W.; Kuo, L. C.; Yim, M. B.; Wells, G. B.; Fukuyama, J. M.; Kim, J. E. J. Am. Chem. Soc. 1985, 107, 5245-5255.

${ }^{46}$ Prescott, J. M.; Wilkes, S. H. Methods Enzymol. 1976, 45B, 530-543.

${ }^{47}$ Chen, G.; Edwards, T.; D'souza, V. M.; Holz, R. C. Biochemistry 1996, in press.

${ }^{48}$ Schalk, C.; Remy, J.-M.; Chevrier, B.; Moras, D.; Tarnus, C. Arch. Biochem. Biophys. 1992, 294, 91-97.

${ }^{49}$ Baker, J. O.; Wilkes, S. H.; Bayliss, M. E.; Prescott, J. M. Biochemistry 1983, 22, 2098-2103.

${ }^{50}$ Prescott, J. M.; Wilkes, S. H.; Wagner, F. W.; Wilson, K. J. J. Biol. Chem. 1971, 246, 1756-1764.

${ }^{51}$ Gill, S. C.; von Hippel, P. H. Anal. Biochem. 1989, 182, 319-326.

${ }^{52}$ Gill, S. C.; von Hippel, P. H. Anal. Biochem. 1990, 189, 283.

${ }^{53}$ Edelhoch, H. Biochemistry 1967, 6, 1948-1954.

${ }^{54}$ Bennett, B.; Berks, B. C.; Ferguson, S. J.; Thomson, A. J.; Richardson, D. J. Eur. J. Biochem. 1994, 226, 789-798.

${ }^{55}$ Bennett, B.; Gruer, M.; Guest, J. R.; Thomson, A. J. Eur. J. Biochem. 1995, 233, 317-326.

${ }^{56}$ Sears, H. J.; Bennett, B.; Spiro, S.; Thomson, A. J.; Richardson, D. J. Biochem. J. 1995, 310, 311-314.

${ }^{57}$ Bennett, B.; Benson, N.; McEwan, A. G.; Bray, R. C. Eur. J. Biochem. 1994, $225,321-331$.

${ }^{58}$ Bray, R. C. Quart. Rev. Biophys. 1988, 21, 299-329.

${ }^{59}$ Belford, R. L. EPR Symposium, 21st Rocky Mountain Conference, Denver, Colorado 1979,

60Maurice, A. M. Ph.D. Thesis, University of Illinois, 1980.

${ }^{61}$ Nilges, M. J. Ph.D. Thesis, University of Illinois, 1979.

${ }^{62}$ Pilbrow, J. R. J. Magn. Res. 1978, 31, 479-489.

${ }^{63}$ Chevrier, B.; D'Orchymont, H.; Schalk, C.; Tarnus, C.; Moras, D. Eur. J. Biochem. 1996, 237, 393-398.

${ }^{64}$ Hagen, W. R. Adv. Inorg. Chem. 1982, 38, 165-222.

${ }^{65}$ Hendrich, M. P.; Debrunner, P. G. Biophys. J. 1989, 56, 489-506.

${ }^{66}$ Munck, E.; Surerus, K. K.; Hendrich, M. P. Metallobiochemistry, Pt D 1993, 227, 463-479.

${ }^{67}$ Hagen, W. R.; Dunham, W. R.; Sands, R. H.; Shaw, R. W.; Beinert, H. Biochim. Biophys. Acta 1984, 765, 399-402. 
${ }^{68}$ Calhoun, M. W.; Gennis, R. B.; Salerno, J. C. FEBS Lett. 1992, 309, 127-129.

${ }^{69}$ Tsubaki, M.; Mogi, T.; Anraku, Y.; Hori, H. Biochemistry 1993, 32, 6065-6072.

${ }^{70}$ Hagen, W. R.; Dunham, W. R.; Johnson, M. K.; Fee, J. A. Biochim. Biophys. Acta 1985, 828, 369-374.

${ }^{71}$ Lynch, J. B.; Juarez-Garcia, C.; Munck, E.; Que, L., Jr. J. Biol. Chem. 1989, 264, 8091-8096.

${ }^{72}$ Verhagen, F. J. M.; Voorhorst, W. G. B.; Kolkman, J. A.; Wolbert, R. B. G.; Hagen, W. R. FEBS Lett. 1993, 336, 13-18.

73Pierik, A. J.; Wassink, H.; Haaker, H.; Hagen, W. R. Eur. J. Biochem. 1993, 212, 51-61.

${ }^{74}$ Sureras, K. K.; Hendrich, M. P.; Christie, P. D.; Rottgardt, D.; OrmeJohnson, W. H.; Munck, E. J. Am. Chem. Soc. 1992, 114, 8579-8590.

${ }^{75}$ Pierik, A. J.; Hagen, W. R.; Dunham, W. R.; Sands, R. H. Eur. J. Biochem. 1992, 206, 705-719.

${ }^{76} \mathrm{Holz}$, R. C.; Brink, J. M.; Gobena, F. T.; O'Connor, C. J. Inorg. Chem. 1994, 33, 6086-6092.

${ }^{77}$ Christianson, D. W.; Lipscomb, W. N. Acc. Chem. Res. 1989, 22, 62-69.

Journal of the American Chemical Society, Vol 119, No. 8 (February 26, 1997): pg. 1923-1933. DOI. This article is (C) American Chemical Society and permission has been granted for this version to appear in e-Publications@Marquette. American Chemical Society does not grant permission for this article to be further copied/distributed or hosted elsewhere without the express permission from American Chemical Society. 